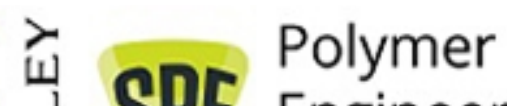 Engineering \& Science
}

\section{Magnetic and Conducting Composites of Cobalt Ferrite Nanorods in a Polyaniline Matrix}

\begin{tabular}{|c|c|}
\hline Journal: & Polymer Engineering \& Science \\
\hline Manuscript ID & PES-19-0186.R2 \\
\hline Wiley - Manuscript type: & Research Article \\
\hline $\begin{array}{r}\text { Date Submitted by the } \\
\text { Author: }\end{array}$ & $n / a$ \\
\hline Complete List of Authors: & $\begin{array}{l}\text { García-Saggión, Nicolás; Universidad de Buenos Aires Facultad de } \\
\text { Ciencias Exactas y Naturales, Química Inorgánica/INQUIMAE } \\
\text { Antonel, Paula; Universidad de Buenos Aires Facultad de Ciencias } \\
\text { Exactas y Naturales, Química Inorgánica/INQUIMAE } \\
\text { Molina, Fernando; Universidad de Buenos Aires Facultad de Ciencias } \\
\text { Exactas y Naturales, Química Inorgánica/INQUIMAE }\end{array}$ \\
\hline Keywords: & conducting polymers, composites, nanoparticles \\
\hline
\end{tabular}


1 Magnetic and Conducting Composites of Cobalt Ferrite Nanorods in a Polyaniline Matrix

3

$4 \quad$ N. A. García Saggión ${ }^{1}$, P. S. Antonel ${ }^{1}$, F. V. Molina* 1

$5 \quad{ }^{1}$ Instituto de Química Física de Materiales, Ambiente y Energía (INQUIMAE),

6 Facultad de Ciencias Exactas y Naturales, Universidad de Buenos Aires, Ciudad

7 Universitaria, Pabellon II, piso 1, C1428EGA, Buenos Aires, Argentina.

CORRESPONDING AUTHOR: Fernando V. Molina. Instituto de Química Física de

Materiales, Ambiente y Energía (INQUIMAE), Facultad de Ciencias Exactas y

Naturales, Universidad de Buenos Aires. Ciudad Universitaria, Pabellon II, piso 1,

C1428EHA Buenos Aires, Argentina. fmolina@qui.fcen.uba.ar

RUNNING TITLE: Cobalt ferrite nanorods-polyaniline composites

ACKNOWLEDGMENTS

The authors gratefully acknowledge funding from the Universidad de Buenos Aires (grant 20020170100249BA), the Consejo Nacional de Investigaciones Científicas y Técnicas (grant PIP F57269) and the Agencia Nacional de Promoción Científica y Tecnológica (grant PICT $2014 \mathrm{~N}^{\mathrm{o}}$ 2289), all of Argentina. P. S. A. and F. V. M. are members of the Carrera del Investigador Científico of CONICET. 


\section{ABSTRACT}

27 Composites of cobalt ferrite nanorods in a polyaniline matrix have been synthesized and characterized by electron microscopy observation, X-ray diffraction, IR spectroscopy,

29 thermogravimetric analysis, electrical conductivity and DC magnetization measurements. The composites were prepared using dodecylbenzenesulphonic acid both as a particle protector and as acid media. In the magnetic experiments hysteresis loops were observed, revealing ferromagnetism for both particles and composites. The results indicate that the magnetic properties of the particles were preserved in the composites, and on the other hand the conductivity was almost independent on the polymer/particle ratio. These composites are new materials which show easily tunable magnetic properties, and are expected to be candidates for applications such as microwave shields.

KEYWORDS: Conducting polymers, composites, nanoparticles. 
44 Composites formed by magnetic nanoparticles (MNPs) embedded in a conducting polymer matrix are very interesting due to the capability of combine electrical conduction with magnetic properties [1-4]. MNPs are very interesting materials, due to their many potential applications [5-8]. Among the materials which have been investigated iron, iron oxides $[9,10]$ and ferrites [11-13] have aroused a great interest. Particularly cobalt ferrite $\left(\mathrm{CoFe}_{2} \mathrm{O}_{4}\right)$ is very interesting because it is a hard magnetic material, showing ferromagnetism at room temperature, having high coercivity and moderate saturation magnetization; in addition, it displays good chemical stability $[14,15]$. Most research has been concerned with small, spherical nanoparticles which fall in the monodomain regime [16]. However, other nanostructures such as nanorods, nanotubes, etc. are also interesting due to the possibility of tuning the magnetic properties by changing the morphology of the material [17-19]. It has been noted that nanorods are interesting candidates for new applications [20], albeit they have not been studied as extensively as anisotropic particles. Consequently, we have investigated here a nanorod shaped material.

Conducting polymers have been intensively studied due to their outstanding chemical, mechanical and optical properties [21-25]. Polyaniline (PANI) is an intensively studied conducting polymer. It is easily synthesized by either chemical or electrochemical routes [26-29], and has been proposed for a high number of different applications [3032]. Many PANI based composites have been proposed [3,13,33-36]. Use of conducting polymers results in materials with properties difficult to obtain with only the individual components, due to the high magnetic susceptibilities and the appreciable electrical conductivity [37-39]. These magnetic composites belong to a new type of multifunctional materials combining properties of ordinary polymers and magnetic 
materials. These materials have been proposed for many applications [40-42]; among them, several studies have been addressed to microwave shielding [43-45]. All these applications reveal the importance of studying these materials from both applied and fundamental points of view.

In this work, composites of $\mathrm{CoFe}_{2} \mathrm{O}_{4}$ nanorods in a PANI matrix have been prepared with in situ aniline polymerization; albeit similar to other materials proposed previously $[3,4]$, composites of cobalt ferrite nanorods in PANI have not been studied before; also, a simple preparation route is shown. The nanorods were prepared in several conditions to select those with better magnetic properties relative to its size, so as to enhance the magnetic properties of the final material. The nanoparticles were characterized by XRD studies, SEM observation, BET surface area measurement and DC magnetization measurements. The composite synthesis was performed in the presence of dodecylbenzenesulfonic acid (DBSA) as both particle protecting agent and acidic media. The composites were characterized by SEM observation, XRD studies, thermogravimetric analysis, electrical conductivity measurements, FTIR spectroscopy and DC magnetization measurements.

\section{MATERIALS AND METHODS}

AR grade chemicals, supplied by Merck and Sigma Aldrich, and water of high purity from a Milli-Q system were employed throughout. Aniline (Ani), was used untreated shortly after being received.

Synthesis of $\mathrm{CoFe}_{2} \mathrm{O}_{4}$ nanorods. 
$91 \mathrm{CoFe}_{2} \mathrm{O}_{4}$ nanorods were prepared by a two-step synthesis method based on Yao et al 92 [46]. In the first step an oxalate precursor $\left[\left(\mathrm{CoFe}_{2}\right)_{1 / 3} \mathrm{C}_{2} \mathrm{O}_{4} \cdot 2 \mathrm{H}_{2} \mathrm{O}\right]$ was synthesized by

\section{Synthesis of $\mathrm{CoFe}_{2} \mathrm{O}_{4}$-PANI composites}

The synthesis of the composites was performed following previous work [47]. Several samples were characterized by the molar ratio:

$r=\frac{n_{A n i}}{n_{\mathrm{CoFe}_{2} \mathrm{O}_{4}}}$

where $n_{A n i}$ and $n_{\mathrm{CoFe}_{2} \mathrm{O}_{4}}$ stand for the mole numbers of aniline and $\mathrm{CoFe}_{2} \mathrm{O}_{4}$ respectively. In the composite preparation the molar ratio in the synthesis, $r_{S}$, was varied between 2.0 and 32.0. 
114 As a first step a suspension is prepared adding an amount of $\mathrm{CoFe}_{2} \mathrm{O}_{4}$ nanorods in 25.0

115

116

117

$d=\frac{K \lambda}{\beta \cos \theta}$

$\mathrm{mL}$ of $0.2 \mathrm{M}$ dodecylbenzenesulfonic acid (DBSA) solution, keeping a $\mathrm{CoFe}_{2} \mathrm{O}_{4}$ :DBSA molar ratio of 0.033 . The system was maintained under ultrasound treatment and strong stirring for $1 \mathrm{~h}$. Then aniline monomer was added according to the desired $r$ ratio and the suspension was kept in the same conditions for another hour. Finally, $25.0 \mathrm{~mL}$ of an ammonium persulfate solution (APS) was added dropwise during $1.5 \mathrm{~h}$ at a constant rate, assuring a molar ratio of 1:1 with respect to the monomer. The resulting reaction mixture was maintained under ultrasound treatment and stirring for $1.5 \mathrm{~h}$. The product was demulsified with $50 \mathrm{~mL}$ of isopropyl alcohol. The precipitate was separated by centrifugation at 5000-10000 $\mathrm{g}$ for 10 minutes and washed thoroughly with Milli-Q water; finally, it was washed with ethanol to remove reactants and oligomers. The obtained pellets were dried at room temperature for $24 \mathrm{~h}$.

\section{X-Ray Diffraction}

The crystalline structure of the particles was studied by X-Ray Diffraction (XRD). The analysis were performed with a Siemens D5000 powder diffractometer using $\mathrm{Cu} \mathrm{K}_{\alpha}$ radiation $(\lambda=1.54056 \AA)$. The average crystallite size was obtained with the aid of the Scherrer equation:

where $K$ is the shape factor, taken here as $0.9, \beta$ is the peak full width at half maximum and $\theta$ is the corresponding Bragg angle. 
137 The particle morphology, size and surface characteristics were studied by Scanning 138 Electron Microscopy (SEM). A Carl Zeiss Supra 40 Gemini field emission microscope was employed, equipped with a secondary electron detector inside the electron column (InLens), a four-quadrant solid-state detector (QBSD, Oxford Instruments, which collects backscattered electrons scattered under very low angle) and an Energy Dispersive X-Ray Spectroscopy (EDS). The samples were prepared by suspending a small amount of each solid in ethanol and approximately 10-15 $\mu \mathrm{L}$ suspension was dropped on a silicon substrate. Particle dimensions were measured employing the ImageJ software, measuring about 100 particles of each sample.

\section{Magnetization measurements}

Magnetization curves were measured at room temperature using a Lakeshore 7400 vibrating sample magnetometer (VSM). Between 10 and $20 \mathrm{mg}$ of each sample were packed with a Teflon tape.

Surface area measurements

Surface area measurement through nitrogen adsorption-desorption isotherms analyzed with the BET theory were performed using a Micrometrics ASAP 2020 system. The $\mathrm{CoFe}_{2} \mathrm{O}_{4}$ samples were degased at $60^{\circ} \mathrm{C}$ for $12 \mathrm{~h}$ and the analysis bath temperature was set on $-195.8^{\circ} \mathrm{C}$ 
160

161

162

163

164

165

166

167

168

169

170

171

172

173

175

176

177

178

179

180

polymer were performed with a FTIR Nicolet 8700 spectrometer, recording spectra in the range $400-4000 \mathrm{~cm}^{-1} .0 .5 \mathrm{mg}$ of each sample was pressed into a pellet with $150 \mathrm{mg}$ of $\mathrm{KBr}$. For each sample, 32 scans were accumulated.

\section{Thermogravimetric analysis}

Thermogravimetric analysis (TGA) of $\mathrm{CoFe}_{2} \mathrm{O}_{4}$ nanorods, PANI and $\mathrm{CoFe}_{2} \mathrm{O}_{4}$-PANI composites was performed with a thermobalance TG-DTA 50 Simultaneous Shimadzu. The thermograms were recorded for $1-5 \mathrm{mg}$ of each sample at a heating rate of $10^{\circ} \mathrm{C}$ $\min ^{-1}$ in the temperature range of $18-810{ }^{\circ} \mathrm{C}$ under air atmosphere.

\section{Conductivity measurements}

The electrical conductivity of the synthesized composites was measured on pressed circular pellets of $1 \mathrm{~cm}$ diameter using a Teq 04 (S. Sobral, Buenos Aires, Argentina) potentiostat/galvanostat under computer control. A known current was applied to each sample for $60 \mathrm{~s}$ and the potential difference was measured, resulting that the experimental data followed Ohm's law. Finally, the pellet thickness was measured using a caliber.

\section{RESULTS AND DISCUSSION}

\section{Cobalt ferrite particles}


181 Particle size and morphology. The first stage of synthesis yielded a yellow powder of cobalt/iron(II) oxalate $\left(\mathrm{CoFe}_{2}\right)_{1 / 3} \mathrm{C}_{2} \mathrm{O}_{4}$ particles. As it is observed in Fig. 1, the particle morphology is dependent on the PVA content in each synthesis; in the absence of PVA short, nearly cubic particles are obtained, whereas with increasing surfactant content nanorods are found with different sizes and even morphologies. Fig. 1 shows that by means of the PVA concentration the size and morphology of the ferrite particles can be controlled.

After the calcination of the oxalate precursors a black fine powder of $\mathrm{CoFe}_{2} \mathrm{O}_{4}$ was obtained, which is attracted by a magnet. Fig. 2 shows SEM images of the resulting particles. Moreover, it is noticeable the ferrite particles maintained the original shape from their precursor. When polyvinylalcohol was added into the synthesis medium, it directed the growth of the precursor of $\mathrm{CoFe}_{2} \mathrm{O}_{4}$ particles in one preferential direction, so that after calcination nanorods with lengths of 3-5 $\mu \mathrm{m}$ and sections ranging 270-500 $\mathrm{nm}$ were obtained. In the absence of PVA, the final product appeared with a great variety of shapes and sizes.

Fig. 3(a) (and Table 3 later on) shows the particle size results found. Upon increasing the amount of polyvinyl alcohol from $0.5 \%$ to $3.0 \%$ sharper rods were obtained, attaining minimum section and length at the latter concentration. When PVA content is further increased, both length and section increase. At $3.0 \%$ PVA (Fig. 3(b)) the ratio of alcohol hydroxyl groups to metal cation concentration is close to unity; this suggests that such ratio is optimal in order to obtain thinner nanorods.

Closer inspection of the final particles (Fig. 4) reveals differences in morphology when compared with the precursor $\left(\mathrm{CoFe}_{2}\right)_{1 / 3} \mathrm{C}_{2} \mathrm{O}_{4}$ particles. The final particles (Fig. 4(b)) show a surface with holes or cavities on the surface, result of an irregular structure with 
solid $\mathrm{CoFe}_{2} \mathrm{O}_{4}$ regions having size in the range of tens of nanometers, held together by thin solid bridges. This morphology is presumably caused by the $\mathrm{CO}_{2}$ released during the calcination phase. The BET surface area of the FINAL $\mathrm{CoFe}_{2} \mathrm{O}_{4}$ particles was 15.9 $\mathrm{m}^{2} \mathrm{~g}^{-1}$

Crystalline structure. XRD results are shown in Fig. 5, where characteristic diffractograms of samples of $\mathrm{CoFe}_{2} \mathrm{O}_{4}$ produced in presence of different PVA concentrations are presented.

As a general remark, all results show that the synthesis produced particles with good crystallinity. The oxalates show the typical orthorhombic phase $[48,49]$ and upon calcinating process they adopt the cubic spinel structure [50], consistent with ferrite crystals. The lattice parameters and crystallite sizes estimated through Scherrer equation using the (311) peak are summarized in Table 1, which lie in the range found in the literature for this material $[17,50]$. It is worth notirng that with the same lattice parameter crystallite sizes vary between 27 and $37 \mathrm{~nm}$ approximately.

Magnetic properties. The curves of magnetization, $M$, as a function of magnetic field, $H$, for all $\mathrm{CoFe}_{2} \mathrm{O}_{4}$ nanorod samples at room temperature are shown in Fig. 6; Fig. 6 (a) presents the original curves for different PVA content in the synthesis medium, and Fig. 6 (b) shows the normalized magnetization, $M / M_{s}$, where $M_{s}$ is the saturation magnetization (taken as the maximum value achieved for positive fields).

Ferromagnetic behavior is observed in all cases. It is found that the coercive field, $H_{C}$, shows little change for all curves whereas the remanence, $M_{r}$, is dependent on the PVA content; the results found, along with literature reports for other types of nanoparticles, are collected in Table 2. Comparing the $H_{C}$ values found here with reported values for other $\mathrm{CoFe}_{2} \mathrm{O}_{4}$ nanoparticles and nanostructures, a noticeable increase is observed, 
except for those obtained through a combustion method, which results in particles of irregular shape and a wide range of sizes [51]; for spherical or quasi-spherical $12 \mathrm{~nm}$ nanoparticles the reported $H_{C}$ values are between $1 / 3$ and $1 / 2$ approximately of the present results. Bulk values are also lower: literature results range from about 600 [52] to $750-1000$ Oe [53]. The high values of the coercive field obtained in this work compared with spherical particles suggests the presence of different mechanisms behind the reversal magnetization; small nanoparticles are in the single-ferromagnetic domain regime, giving place to coherent spin rotation as the mechanism governing the magnetization reversal. In the present case, the nanorods are built from several nanorods in Fig. 4(b) appear to be constituted of quasi spherical grains bound together by thinner bridges. SEM image analysis was performed employing ImageJ software to estimate grains sizes. The results show that considering the grains as spheroids, the average major axis length is about $130 \mathrm{~nm}$ while the minor axis measures about $67 \mathrm{~nm}$; thus, these grains are not formed by single crystallites. The high $H_{C}$ values suggest that there are strong spin interactions between crystallites in grains. Consistently, as it is observed in Table 3, rods with narrower sections show lower coercive field values. Likewise, other nanorods and nanotubes found in literature have also higher $H_{C}$ than spherical particles.

Considering the saturation magnetization, it is found that the narrower nanorods show higher values. In fact the $\mathrm{CoFe}_{2} \mathrm{O}_{4}$ nanorods synthesized with 3.0 and $4.0 \%$ PVA show $M_{S}$ values of 53-54 emu $\mathrm{g}^{-1}$, while all other samples have values not higher than $48 \mathrm{emu}$ $\mathrm{g}^{-1}$. Comparing the $M_{S}$ values of the nanorods of smaller sections in this work with the nanotubes and nanorods already cited [17], similar results are found. Also, spherical NPs show in most cases similar values, except for the smaller $12 \mathrm{~nm}$ particles $(65 \mathrm{emu}$ 
$254 \mathrm{~g}^{-1}$ ), where clearly a size effect is present. On the other hand, bulk $M_{S}$ values are

255

256

257

258

259

260

261

262

263

264

265

266

267

268

269

270

271

272

273

274

275

276 generally higher, about 77-83 emu g-1 $[52,53]$.

Regarding the remanence ratio $M_{r} / M_{S}$, it is observed (Fig. 6(b)) that for PVA synthesis concentrations of $3-4 \%$, this parameter is close to 0.5 , whereas for the other cases it is lower, in the range $0.35-0.40$. All other nanoparticles in Table 3 have also low values, and this is generally found with $\mathrm{CoFe}_{2} \mathrm{O}_{4}$ nanoparticles [54].

Considering the results found for the properties of cobalt ferrite nanorods, those obtained with $3.0 \%$ PVA were selected for composite synthesis, due to their smaller section and higher $M_{S}$.

\section{$\mathrm{CoFe}_{2} \mathrm{O}_{4}-\mathrm{PANI}$ composites}

Thermal analysis and composition. The synthesized $\mathrm{CoFe}_{2} \mathrm{O}_{4}-\mathrm{PANI}$ composites were bright emerald green powders, consistent with literature [3,13]. TG analysis was performed to obtain the experimental composition data for all $\mathrm{CoFe}_{2} \mathrm{O}_{4}-\mathrm{PANI}$ composites. In Fig. 7 several thermograms are presented, which are consistent with similar materials $[3,13]$. It is found that PANI is completely decomposed at the final temperature, while cobalt ferrite particles suffer only a small mass loss, due to loss of residual water. In between those results lay the observations for all $\mathrm{CoFe}_{2} \mathrm{O}_{4}$-PANI composites samples, confirming its composition. All curves in Fig. 8 show an initial mass loss until $150{ }^{\circ} \mathrm{C}$ that is attributable to the removal of water molecules from the materials. The onset of polyaniline decomposition is observed at $\sim 250-300{ }^{\circ} \mathrm{C}$, to reach its end at approximately $600{ }^{\circ} \mathrm{C}$. This point is in agreement with literature reports $[3,13,33]$. 
277 Regarding this analysis and results an estimation of the composites composition can be obtained, considering that for temperatures lower than $150{ }^{\circ} \mathrm{C}$ water elimination takes place and that at $800{ }^{\circ} \mathrm{C}$ the remaining mass is only from $\mathrm{CoFe}_{2} \mathrm{O}_{4}$ particles. In doing this estimation, it should be taken into account the presence of DBSA, because it is the only acid present. Considering that the PANI product is obtained in the doped emeraldine form, an average of half the $\mathrm{N}$ atoms are expected be protonated [55]; because the dopant anion is dodecylbencensulfate (DBS), one mole of DBS should be present for each two of aniline monomer, and should be included in the mass loss. Taking this into account, the results for the experimental monomer/ferrite ratio in the products, $r_{P}$, compared to the synthesis composition $r_{S}$ are shown in Table 3 along with the $\mathrm{CoFe}_{2} \mathrm{O}_{4}$ mass fraction computed from $r_{P}$. For comparison, in Table 3 results from a previous study [3] are included. It is observed that the polymer ratio in the product is lower than $r_{S}$; this is attributable to aniline loss due to incomplete polymerization and oligomers removal during product purification.

Morphology. In Fig. 8 SEM images of composites having different $r_{S}$ values are displayed; in a), c), e), f) and g) standard images (InLens detector) are displayed, whereas in b), d) and h) the QBSD detector was employed, which enhances contrast of metallic elements. Fig. 8 a) and b) show that, for $r_{S}=6$, the composite is not well formed and so many particles are partially or totally uncovered, with the polymer growing in between particles. This is attributable to the low polymer content in the composite $\left(r_{P}=0.9\right)$. Fig. $\left.8 \mathrm{~b}\right)$ clearly distinguishes nanorods as white areas revealed by the QBSD detector.

When $r_{S}$ is greater than 6 , the composites show quite different morphologies. For $r_{S}=$ 12 and 16 a globular morphology is observed, whereas for $r_{S}=24$ there is a more fibrous appearance. In all these cases, the morphology consists in a polymeric matrix 
with particles included and dispersed in the matrix. The QBSD images (Fig.8 d) and h)) display dark areas that correspond to conductive polymer matrix and, scattered, clearer spots and bars which correspond to the $\mathrm{CoFe}_{2} \mathrm{O}_{4}$ nanorods. The lower contrast observed in the images is clearly caused by the polymer matrix; this in turn indicates that the particles are embedded in the matrix rather than on the surface. Thus, the morphology of the composites is controlled through $r_{S}$.

These observations suggest that the growth of the conductive polymer could be interpreted by a nucleation effect of aniline monomers on the surface of the nanorods. That would clarify the observations on Figure 8 a) and b) where polyaniline grows only among particles, and the fact that at higher $r_{S}$ values the nanorods become hidden by the polymer matrix evidencing the growth of the latter over the former. This scheme is in agreement with observations made in other reports [3].

IR spectroscopy. The IR spectra of these materials is consistent with literature reports [3]. Here, we will focus in the medium wavenumber region. In Fig. 9 the IR region between $400-1800 \mathrm{~cm}^{-1}$ is plotted; the spectra of $\mathrm{CoFe}_{2} \mathrm{O}_{4}$ particles, PANI polymer and two composites are shown. The IR spectrum of PANI confirms the presence of the main functional groups present in this polymer [56]. The most important bands are highlighted by solid vertical lines: the bands at 1566 and $1479 \mathrm{~cm}^{-1}$ are attributed to the quinonoid/benzenoid ring stretching respectively, the $1292 \mathrm{~cm}^{-1}$ band corresponds to C$\mathrm{N}$ vibration of secondary aromatic amines, at $1112 \mathrm{~cm}^{-1}$ ring-N vibrations and the 784 $\mathrm{cm}^{-1}$ band due to $\mathrm{C}-\mathrm{H}$ out of plane vibration. In Fig. 9 (a) the main lattice band of $\mathrm{CoFe}_{2} \mathrm{O}_{4}$ is marked at $588 \mathrm{~cm}^{-1}$ [4] in dashed trace. Fig. 9 (c) shows the spectrum for the composite with $r_{S}=6\left(r_{P}=0.9\right)$, where it is observed the shift of the ferrite main band to lower wavenumbers. Moreover all main PANI IR bands here studied appear shifted to higher wavenumbers. The other composite considered $\left(r_{S}=16, r_{P}=8.6\right)$ is shown in 
327 Fig. 9 (d), where the particle main lattice band is shifted to higher energy values. It is less noticeable than for the $r_{P}=0.9$ composite, as expected since the latter is composed by a larger proportion of particles. Besides, the bands assigned to polyaniline are visible and shifted to higher wave numbers compared with the pure polymer. Moreover in Fig.

9 (b), (c) and (d) the most important band of DBS within the region studied is highlighted within a circle at approximately $1004-1029 \mathrm{~cm}^{-1}$ [4]. This proves that this anion is present in the final products.

The results reported here show that the main bands from the PANI appear in the composites IR shifted towards higher wavenumber values. On the other hand the main lattice band coming from the ferrite particles are also visible in the composite spectra and suffer a shifting to higher energies in this case. This evidence suggests the presence of interactions in the composite matrix between PANI, the $\mathrm{CoFe}_{2} \mathrm{O}_{4}$ particles and, presumably the DBS anions. Further analysis of PANI structure can be done by observing the ring deformation bands position. Upon going from the undoped PANI base to the fully doped salt form there is a visible red shift of the aforementioned bands. In Table 4 the positions of the ring deformation bands obtained in this work and bibliography data are presented.

Electrical conductivity. The electrical conductivity of pure polyaniline and $\mathrm{CoFe}_{2} \mathrm{O}_{4}$ PANI composites prepared in this work (Table 5) shows lower values than those expected form bibliography for PANI [57], ranging between $6.9 \times 10^{-5} \mathrm{~S} \mathrm{~cm}^{-1}$ and $1.8 \times$ $10^{-4} \mathrm{~S} \mathrm{~cm}^{-1}$, not showing a definite tendency with composition. The only exception is the composite with lower polymer content $\left(r_{S}=6\right)$ for which the resistance was almost infinity; this fact can be explained by the low, fragmentary, polymer content in the product. For all the other composites, the low conductivity can be explained by synthesis conditions, more specifically by the low aniline concentration in the medium 
352 leading to the formation of short polymer chains [58]. The lack of conductivity change with material composition can be attributed to PANI percolating efficiently in all cases, due to relatively high polymer contents.

Albeit decomposition does not start up to $\sim 250{ }^{\circ} \mathrm{C}$, as seen in Fig. 7, the resistivity increases irreversibly upon heating, reaching one order of magnitude higher after heating to $\sim 90{ }^{\circ} \mathrm{C}$ for 1 hour (Table 5), thus there is relatively low temperature stability, even when PANI itself is stable. Presumably, DBSA undergoes some transformation which affects the polymer protonation state and/or structure, decreasing conductivity.

Magnetic properties. Magnetization curves as function of applied field measured for the $\mathrm{CoFe}_{2} \mathrm{O}_{4}-\mathrm{PANI}$ composites are presented in Fig. 10. All curves display a hysteresis loop, demonstrating that the composites maintain the typical ferromagnetic behavior of the $\mathrm{CoFe}_{2} \mathrm{O}_{4}$ particles. The results displayed in Fig. 10 (a) for the magnetization as a function of the total mass of the composite show that in general the magnetization decreases when the relative amount of ferrites in the composite decreases, as expected.

On the other hand, in Fig. 10 (b) the magnetization is referred to the actual ferrite mass present in each composite as estimated by TGA analysis (Table 3); for comparison, the hysteresis loop for the bare particles is shown. It is observed that with this normalization all the composite samples reach $M_{S}$ values close to the value of the starting particles. in the composites than the bare particles. Also, there are small decreases in the remanence of the composites compared with the $\mathrm{CoFe}_{2} \mathrm{O}_{4}$ nanorods. Overall, however,

373 it can be concluded that the particles magnetic behavior is essentially preserved in the composites, and that the material magnetization is an almost linear function of the 
376 microwave shields $[13,35]$, thus the composites studied in this work could be proposed

377 for shielding applications.

Regarding temperature dependence of the magnetic properties, the conductivity changes upon heating reported above prevented further investigation. However, the magnetic properties are expected to be stable in the room temperature range, because such properties come from the cobalt ferrite nanorods, a material whose magnetism is known to be stable around room temperature.

\section{CONCLUSIONS}

In this work, novel cobalt ferrite nanorod based composites having both ferromagnetic and electrically conducting behavior are obtained. First, the synthesis of cobalt ferrite nanorods using a simple synthesis assisted by polyvinyl alcohol as surfactant is demonstrated. The ratio of surfactant to cation concentration was optimized in order to improve the magnetization and reduce the size of the nanorods. The composites prepared with these nanorods in a polyaniline matrix show that the magnetic properties of the particles are preserved, and the magnetic response of the material is easily tuned through the ferrite content. On the other hand, except for very low polymer content, the conductivity of the composites is nearly independent of the ferrite content, thus the magnetic behavior can be adjusted without affecting the electrical conductivity. 


\section{REFERENCES}

397

1. Murillo, N., Ochoteco, E., Alesanco, Y., Pomposo, J.A., Rodriguez, J., Gonzalez, J., Del Val, J.J., Gonzalez, J.M., Britel, M.R., Varela-Feria, F.M., and others (2004) CoFe2O4-polypyrrole (PPy) nanocomposites: new multifunctional materials. Nanotechnology, 15 (4), S322.

2. Dar, M.A., Kotnala, R.K., Verma, V., Shah, J., Siddiqui, W.A., and Alam, M. (2012) High Magneto-Crystalline Anisotropic Core-Shell Structured Mn0.5Zn0.5Fe2O4/Polyaniline Nanocomposites Prepared by in Situ Emulsion Polymerization. J. Phys. Chem. C, 116 (9), 5277-5287.

3. Antonel, P.S., Berhó, F.M., Jorge, G., and Molina, F.V. (2015) Magnetic composites of $\mathrm{CoFe} 2 \mathrm{O} 4$ nanoparticles in a poly(aniline) matrix: Enhancement of remanence ratio and coercivity. Synthetic Metals, 199, 292-302.

4. Muñoz Resta, I., Sellés, J.M., Lanús-Méndez-Elizalde, M., Antonel, P.S., and Molina, F.V. (2017) Polypyrrole-CoFe ${ }_{2} \mathrm{O}_{4}$ nanocomposites: Polymer influence on magnetic behavior and particle effects on polymer conduction. Polymer Composites.

5. Leslie-Pelecky, D.L., and Rieke, R.D. (1996) Magnetic Properties of Nanostructured Materials. Chem. Mater., 8 (8), 1770-1783.

6. Kodama, R.H. (1999) Magnetic nanoparticles. J. Magn. Magn. Mater., 200 (1-3), $359-372$.

7. Chowdhury, S.R., and Yanful, E.K. (2010) Arsenic and chromium removal by mixed magnetite-maghemite nanoparticles and the effect of phosphate on removal. Journal of Environmental Management, 91 (11), 2238-2247. 
8. Beveridge, J.S., Stephens, J.R., and Williams, M.E. (2011) The Use of Magnetic Nanoparticles in Analytical Chemistry. Annual Review of Analytical Chemistry, 4 (1), 251-273.

9. Jin, J., Hashimoto, K., and Ohkoshi, S. (2005) Formation of spherical and rodshaped ?-Fe2O3 nanocrystals with a large coercive field. J. Mater. Chem., 15 (10), 1067.

10. Tavakoli, A., Sohrabi, M., and Kargari, A. (2007) A review of methods for synthesis of nanostructured metals with emphasis on iron compounds. Chem. Pap., $61(3), 151-170$.

11. Kim, Y.I., Kim, D., and Lee, C.S. (2003) Synthesis and characterization of $\mathrm{CoFe} 2 \mathrm{O} 4$ magnetic nanoparticles prepared by temperature-controlled coprecipitation method. Physica B, 337 (1-4), 42-51.

12. Mu, G., Pan, X., Chen, N., Gan, K., and Gu, M. (2008) Preparation and magnetic properties of barium hexaferrite nanorods. Mater. Res. Bull., 43 (6), 1369-1375.

13. Khairy, M. (2014) Synthesis, characterization, magnetic and electrical properties of polyaniline/NiFe2O4 nanocomposite. Synthetic Metals, 189, 34-41.

14. Liu, C., Zou, B., Rondinone, A.J., and Zhang, Z.J. (2000) Chemical Control of Superparamagnetic Properties of Magnesium and Cobalt Spinel Ferrite Nanoparticles through Atomic Level Magnetic Couplings. J. Am. Chem. Soc., 122 (26), 6263-6267.

15. Mazarío, E., Herrasti, P., Morales, M.P., and Menéndez, N. (2012) Synthesis and characterization of CoFe2O4-ferrite nanoparticles obtained by an electrochemical method. Nanotechnology, 23 (35), 355708.

16. O’Handley, R.C. (1999) Modern Magnetic Materials: Principles and Applications, Wiley-Interscience, New York. 
17. Antonel, P.S., Oliveira, C.L.P., Jorge, G.A., Perez, O.E., Leyva, A.G., and Negri, R.M. (2015) Synthesis and characterization of CoFe2O4 magnetic nanotubes, nanorods and nanowires. Formation of magnetic structured elastomers by magnetic field-induced alignment of CoFe2O4 nanorods. J Nanopart Res, 17 (7), 294.

18. Chen, Y.-J., Xiao, G., Wang, T.-S., Ouyang, Q.-Y., Qi, L.-H., Ma, Y., Gao, P., Zhu, C.-L., Cao, M.-S., and Jin, H.-B. (2011) Porous Fe3O4/Carbon Core/Shell Nanorods: Synthesis and Electromagnetic Properties. J. Phys. Chem. C, 115 (28), $13603-13608$.

19. Zhang, S., Xu, W., Zeng, M., Li, J., Li, J., Xu, J., and Wang, X. (2013) Superior adsorption capacity of hierarchical iron oxide@magnesium silicate magnetic nanorods for fast removal of organic pollutants from aqueous solution. J. Mater. Chem. A, 1 (38), 11691-11697.

20. Hore, M.J.A., and Composto, R.J. (2014) Functional Polymer Nanocomposites Enhanced by Nanorods. Macromolecules, 47 (3), 875-887.

21. Chandrasekhar, P. (1999) Conducting Polymers, Fundamentals and Applications: A Practical Approach, Springer, New York.

22. Lizarraga, L., Andrade, E.M., and Molina, F.V. (2004) Swelling and volume changes of polyaniline upon redox switching. J. Electroanal. Chem., 561 (SUPPL. 1), 127-135.

23. Skotheim, T.A., and Reynolds, J. (eds.) (2006) Conjugated Polymers: Processing and Applications, CRC Press, Boca Raton, Fl, USA.

24. Antonel, P.S., Völker, E., and Molina, F.V. (2012) Photophysics of polyaniline: Sequence-length distribution dependence of photoluminescence quenching as studied by fluorescence measurements and Monte Carlo simulations. Polymer, $\mathbf{5 3}$ (13), 2619-2627. 
25. Wen, J., Tan, X., Hu, Y., Guo, Q., and Hong, X. (2017) Filtration and Electrochemical Disinfection Performance of PAN/PANI/AgNWs-CC Composite Nanofiber Membrane. Environ. Sci. Technol., 51 (11), 6395-6403.

26. Huang, W.-S., Humphrey, B.D., and MacDiarmid, A.G. (1986) Polyaniline, a novel conducting polymer. Morphology and chemistry of its oxidation and reduction in aqueous electrolytes. J. Chem. Soc., Faraday Trans. 1, 82 (8), 2385-2400.

27. Kang, E.T., Neoh, K.G., and Tan, K.L. (1998) Polyaniline: A polymer with many interesting intrinsic redox states. Prog. Polym. Sci., 23 (2), 277-324.

28. Lee, K., Cho, S., Park, S.H., Heeger, A.J., Lee, C.-W., and Lee, S.-H. (2006) Metallic transport in polyaniline. Nature, 441 (7089), 65-68.

29. Bhadra, S., Khastgir, D., Singha, N.K., and Lee, J.H. (2009) Progress in preparation, processing and applications of polyaniline. Progress in Polymer Science, 34 (8), 783-810.

30. McQuade, D.T., Pullen, A.E., and Swager, T.M. (2000) Conjugated Polymer-Based Chemical Sensors. Chemical Reviews, 100 (7), 2537-2574.

31. Guan, H., Fan, L.-Z., Zhang, H., and Qu, X. (2010) Polyaniline nanofibers obtained by interfacial polymerization for high-rate supercapacitors. Electrochim. Acta, $\mathbf{5 6}$ (2), 964-968.

32. Conzuelo, L.V., Arias-Pardilla, J., Cauich-Rodríguez, J.V., Smit, M.A., and Otero, T.F. (2010) Sensing and Tactile Artificial Muscles from Reactive Materials. Sensors, 10 (4), 2638-2674.

33. Wang, S., Tan, Z., Li, Y., Sun, L., and Zhang, T. (2006) Synthesis, characterization and thermal analysis of polyaniline/ZrO2 composites. Thermochimica Acta, 441 (2), $191-194$. 
34. Hatchett, D.W., and Josowicz, M. (2008) Composites of Intrinsically Conducting Polymers as Sensing Nanomaterials. Chem. Rev., 108 (2), 746-769.

35. Gandhi, N., Singh, K., Ohlan, A., Singh, D.P., and Dhawan, S.K. (2011) Thermal, dielectric and microwave absorption properties of polyaniline- $\mathrm{CoFe} 2 \mathrm{O} 4$ nanocomposites. Compos. Sci. Technol., 71 (15), 1754-1760.

36. Ćirić-Marjanović, G. (2013) Recent advances in polyaniline composites with metals, metalloids and nonmetals. Synthetic Metals, 170, 31-56.

37. Gangopadhyay, R., and De, A. (2000) Conducting Polymer Nanocomposites: A Brief Overview. Chem. Mater., 12 (3), 608-622.

38. Pyun, J. (2007) Nanocomposite Materials from Functional Polymers and Magnetic Colloids. Polym. Rev., 47 (2), 231-263.

39. Singh, K., Ohlan, A., Bakhshi, A.K., and Dhawan, S.K. (2010) Synthesis of conducting ferromagnetic nanocomposite with improved microwave absorption properties. Mater. Chem. Phys., 119 (1-2), 201-207.

40. Radhakrishnan, S., Prakash, S., Rao, C.R.K., and Vijayan, M. (2009) Organically Soluble Bifunctional Polyaniline-Magnetite Composites for Sensing and Supercapacitor Applications. Electrochemical and Solid-State Letters, 12 (4), A84.

41. Zhu, J., Wei, S., Zhang, L., Mao, Y., Ryu, J., Karki, A.B., Young, D.P., and Guo, Z. (2011) Polyaniline-tungsten oxide metacomposites with tunable electronic properties. J. Mater. Chem., 21 (2), 342-348.

42. Xiong, P., Chen, Q., He, M., Sun, X., and Wang, X. (2012) Cobalt ferritepolyaniline heteroarchitecture: a magnetically recyclable photocatalyst with highly enhanced performances. Journal of Materials Chemistry, 22 (34), 17485.

43. Qiao, M., Lei, X., Ma, Y., Tian, L., Su, K., and Zhang, Q. (2016) Well-Defined Core-Shell Fe3O4@Polypyrrole Composite Microspheres with Tunable Shell 
Thickness: Synthesis and Their Superior Microwave Absorption Performance in the Ku Band. Industrial and Engineering Chemistry Research, 55 (22), 6263-6275.

44. Yang, R.-B., Reddy, P.M., Chang, C.-J., Chen, P.-A., Chen, J.-K., and Chang, C.-C. (2016) Synthesis and characterization of Fe3O4/polypyrrole/carbon nanotube composites with tunable microwave absorption properties: Role of carbon nanotube and polypyrrole content. Chemical Engineering Journal, 285, 497-507.

45. Wu, L., Zhang, Q., Hong, J., Dong, Z., and Wang, J. (2019) Degradation of bisphenol A by persulfate activation via oxygen vacancy-rich CoFe2O4-x. Chemosphere, 221, 412-422.

46. Yao, X., Kong, J., Zhao, C., Zhou, D., Zhou, R., and Lu, X. (2014) Zinc ferrite nanorods coated with polydopamine-derived carbon for high-rate lithium ion batteries. Electrochimica Acta, 146, 464-471.

47. Muñoz Resta, I., Horwitz, G., Lanús Mendez Elizalde, M., Jorge, G., Molina, F.V., and Antonel, P.S. (2013) Magnetic and Conducting Properties of Composites of Conducting Polymers and Ferrite Nanoparticles. IEEE T. Magn.

48. Tominaka, S., and Cheetham, A.K. (2014) Intrinsic and extrinsic proton conductivity in metal-organic frameworks. RSC Adv., 4 (97), 54382-54387.

49. López, M.C., Tirado, J.L., and Pérez Vicente, C. (2013) Structural and comparative electrochemical study of M(II) oxalates, M = Mn, Fe, Co, Ni, Cu, Zn. Journal of Power Sources, 227, 65-71.

50. Wyckoff, R.W.G. (1931) The structure of crystals, Chemical Catalog Co., New York.

51. Houshiar, M., Zebhi, F., Razi, Z.J., Alidoust, A., and Askari, Z. (2014) Synthesis of cobalt ferrite (CoFe2O4) nanoparticles using combustion, coprecipitation, and 
precipitation methods: A comparison study of size, structural, and magnetic properties. Journal of Magnetism and Magnetic Materials, 371, 43-48.

52. Dedi, Idayanti, N., Kristiantoro, T., Alam, G.F.N., and Sudrajat, N. (2018) Magnetic properties of cobalt ferrite synthesized by mechanical alloying. AIP Conference Proceedings, 1964 (1), 020003.

53. Maaz, K., Mumtaz, A., Hasanain, S.K., and Ceylan, A. (2007) Synthesis and magnetic properties of cobalt ferrite ( $\mathrm{CoFe} 2 \mathrm{O} 4)$ nanoparticles prepared by wet chemical route. J. Magn. Magn. Mater., 308 (2), 289-295.

54. Geng, B., Ding, Z., and Ma, Y. (2016) Unraveling the correlation between the remanence ratio and the dipolar field in magnetic nanoparticles by tuning concentration, moment, and anisotropy. Nano Res., 9 (9), 2772-2781.

55. Ćirić-Marjanović, G. (2013) Recent advances in polyaniline research: Polymerization mechanisms, structural aspects, properties and applications. Synthetic Metals, 177, 1-47.

56. Trchová, M., and Stejskal, J. (2011) Polyaniline: The infrared spectroscopy of conducting polymer nanotubes (IUPAC Technical Report). Pure and Applied Chemistry, 83 (10), 1803-1817.

57. Salaneck, W.R., Lundström, I., Hjertberg, T., Duke, C.B., Conwell, E., Paton, A., MacDiarmid, A.G., Somasiri, N.L.D., Huang, W.S., and Richter, A.F. (1987) Electronic structure of some polyanilines. Synthetic Metals, 18 (1-3), 291-296.

58. Rakić, A., Bajuk-Bogdanović, D., Mojović, M., Ćirić-Marjanović, G., MilojevićRakić, M., Mentus, S., Marjanović, B., Trchová, M., and Stejskal, J. (2011) Oxidation of aniline in dopant-free template-free dilute reaction media. Mater. Chem. Phys., 127 (3), 501-510. 
566 59. Antonel, P.S., Jorge, G., Perez, O.E., Butera, A., Leyva, A.G., and Negri, R.M.

567 (2011) Magnetic and elastic properties of $\mathrm{CoFe} 2 \mathrm{O} 4-$ polydimethylsiloxane

568 magnetically oriented elastomer nanocomposites. J. Appl. Phys., 110 (4), $0439201-$

569 8.

570

60. Rashidi, S., and Ataie, A. (2013) One-Step Synthesis of $\mathrm{CoFe}_{2} \mathrm{O}_{4}$ Nano-Particles by Mechanical Alloying. Advanced Materials Research, 829, 747-751.

572

61. Kulszewicz-Bajer, I., Sobczak, J., Hasik, M., and Pretula, J. (1996) Spectroscopic

573 studies of polyaniline protonation with poly(alkylene phosphates). Polymer, 37 (1),

574 25-30.

575 
577 Table 1. Lattice parameter $a$ and crystallite size obtained from Fig. 5.

\begin{tabular}{ccc}
\hline sample & Lattice parameter $(\AA)$ & Crystallite size (nm) \\
\hline $\mathrm{CoFe}_{2} \mathrm{O}_{4}$ 0.5\% PVA & $8.36 \pm 0.02$ & $27.71 \pm 0.02$ \\
$\mathrm{CoFe}_{2} \mathrm{O}_{4} 1.0 \%$ PVA & $8.38 \pm 0.02$ & $32.90 \pm 0.04$ \\
$\mathrm{CoFe}_{2} \mathrm{O}_{4} 2.0 \%$ PVA & $8.37 \pm 0.02$ & $32.91 \pm 0.04$ \\
$\mathrm{CoFe}_{2} \mathrm{O}_{4} 3.0 \%$ PVA & $8.37 \pm 0.02$ & $35.09 \pm 0.04$ \\
$\mathrm{CoFe}_{2} \mathrm{O}_{4} 4.0 \%$ PVA & $8.37 \pm 0.02$ & $37.60 \pm 0.05$ \\
$\mathrm{CoFe}_{2} \mathrm{O}_{4} 5.0 \%$ PVA & $8.37 \pm 0.02$ & $27.71 \pm 0.02$ \\
$\mathrm{CoFe}_{2} \mathrm{O}_{4}$ Ref. [50] & 8.35 & - \\
\hline
\end{tabular}

578 
580 Table 2. Magnetic properties for $\mathrm{CoFe}_{2} \mathrm{O}_{4}$ particles of different morphologies.

\begin{tabular}{|c|c|c|c|c|c|}
\hline $\mathrm{CoFe}_{2} \mathrm{O}_{4}$ sample & Size & $\begin{array}{c}M_{S} / \mathrm{emu} \mathrm{g}^{-} \\
1\end{array}$ & $M_{r} / M_{S}$ & $H_{C} / \mathrm{Oe}$ & Ref. \\
\hline nanorods $0.5 \%$ PVA & $\begin{array}{c}4 \mu \mathrm{m} \times 600 \\
\mathrm{~nm}\end{array}$ & 46 & 0.35 & 1518 & $\begin{array}{l}\text { This } \\
\text { work }\end{array}$ \\
\hline nanorods $1.0 \%$ PVA & $\begin{array}{c}5 \mu \mathrm{m} \times 600 \\
\mathrm{~nm}\end{array}$ & 43 & 0.35 & 1484 & $\begin{array}{l}\text { This } \\
\text { work }\end{array}$ \\
\hline nanorods $2.0 \% \mathrm{PVA}$ & $\begin{array}{c}4 \mu \mathrm{m} \times 500 \\
\mathrm{~nm}\end{array}$ & 48 & 0.38 & 1509 & $\begin{array}{l}\text { This } \\
\text { work }\end{array}$ \\
\hline nanorods $3.0 \%$ PVA & $\begin{array}{c}3 \mu \mathrm{m} \times 270 \\
\mathrm{~nm}\end{array}$ & 54 & 0.48 & 1374 & $\begin{array}{l}\text { This } \\
\text { work }\end{array}$ \\
\hline nanorods $4.0 \%$ PVA & $\begin{array}{c}5 \mu \mathrm{m} \times 400 \\
\mathrm{~nm}\end{array}$ & 53 & 0.49 & 1391 & $\begin{array}{l}\text { This } \\
\text { work }\end{array}$ \\
\hline nanorods $5.0 \%$ PVA & $\begin{array}{c}5 \mu \mathrm{m} \times 410 \\
\mathrm{~nm}\end{array}$ & 48 & 0.40 & 1491 & $\begin{array}{l}\text { This } \\
\text { work }\end{array}$ \\
\hline $\begin{array}{l}\text { spherical nanoparticles } \\
\text { (precipitation) }\end{array}$ & $12 \mathrm{~nm}$ & & 0.18 & 400 & [59] \\
\hline $\begin{array}{l}\text { spherical nanoparticles } \\
\text { (mechanical alloying) }\end{array}$ & $50-25 \mathrm{~nm}$ & $47-52$ & $\begin{array}{l}0.28- \\
0.35\end{array}$ & $\begin{array}{l}575- \\
831\end{array}$ & {$[60]$} \\
\hline $\begin{array}{l}\text { spherical nanoparticles } \\
\text { (precipitation) }\end{array}$ & $35 \mathrm{~nm}$ & 47 & 0.28 & 233 & {$[51]$} \\
\hline $\begin{array}{l}\text { spherical nanoparticles } \\
\text { (coprecipitation) }\end{array}$ & $50 \mathrm{~nm}$ & 56 & 0.45 & 850 & {$[51]$} \\
\hline $\begin{array}{c}\text { nanoparticles (irr. shape) } \\
\text { (combustion) }\end{array}$ & $70 \mathrm{~nm}$ & 57 & 0.46 & 2002 & {$[51]$} \\
\hline nanotubes & $\begin{array}{c}4 \mu \mathrm{m} \times 700 \\
\mathrm{~nm}\end{array}$ & 55 & 0.40 & 1100 & {$[17]$} \\
\hline nanorods & $\begin{array}{l}1.3 \mu \mathrm{m} \mathrm{x} \\
142 \mathrm{~nm}\end{array}$ & 53 & 0.40 & 1000 & {$[17]$} \\
\hline
\end{tabular}


583 Table 3. Composites composition estimated from TGA measurements.

\begin{tabular}{ccc}
\hline Synthesis composition & Measured composition & $\mathrm{CoFe}_{2} \mathrm{O}_{4}$ mass fraction \\
$r_{S}$ & $r_{P}$ & $f_{C F}$ \\
\hline 6 & 0.9 & 0.51 \\
8 & 3.7 & 0.20 \\
12 & 6.2 & 0.13 \\
16 & 8.6 & 0.10 \\
24 & 7.3 & 0.11 \\
32 & 17.0 & 0.05 \\
$5 *$ & 5.9 & 0.30 \\
$10^{*}$ & 20.8 & 0.11 \\
$20 *$ & 24.1 & 0.095
\end{tabular}

* From Ref. [3]. 
586 Table 4. IR Ring deformation bands $\left(\mathrm{cm}^{-1}\right)$ in this work and in references.

\begin{tabular}{cccccc}
\hline Band & PANI & $r_{S}=6$ & $r_{S}=16$ & PANI base [61] & PANI salt [61] \\
\hline $\begin{array}{c}\text { Quinonoid ring } \\
\text { stretching }\end{array}$ & 1566 & 1570 & 1566 & 1583 & 1571 \\
$\begin{array}{c}\text { Benzenoid ring } \\
\text { stretching }\end{array}$ & 1479 & 1496 & 1487 & 1493 & 1479 \\
\hline
\end{tabular}


589 Table 5. Electrical conductivities of PANI and composites obtained.

590

\begin{tabular}{cccc}
\hline Material & $r_{S}$ & $T\left({ }^{\circ} \mathrm{C}\right)$ & $\sigma\left(\mathrm{S} \mathrm{cm}^{-1}\right)$ \\
\hline PANI & $\infty$ & 20 & $1.7 \times 10^{-4}$ \\
$\mathrm{CoFe}_{2} \mathrm{O}_{4}$-PANI & 8 & 20 & $2.5 \times 10^{-4}$ \\
$\mathrm{CoFe}_{2} \mathrm{O}_{4}$-PANI & 12 & 20 & $6.9 \times 10^{-5}$ \\
$\mathrm{CoFe}_{2} \mathrm{O}_{4}$-PANI & 16 & 20 & $1.8 \times 10^{-4}$ \\
$\mathrm{CoFe}_{2} \mathrm{O}_{4}$-PANI & 24 & 20 & $1.8 \times 10^{-4}$ \\
$\mathrm{CoFe}_{2} \mathrm{O}_{4}$-PANI & 32 & 20 & $1.5 \times 10^{-4}$ \\
$\mathrm{CoFe}_{2} \mathrm{O}_{4}$-PANI & 24 & 38 & $5.2 \times 10^{-5} *$ \\
$\mathrm{CoFe}_{2} \mathrm{O}_{4}$-PANI & 24 & 55 & $4.6 \times 10^{-5} *$ \\
$\mathrm{CoFe}_{2} \mathrm{O}_{4}$-PANI & 24 & 90 & $1.5 \times 10^{-5} *$
\end{tabular}

591

* Irreversible change, does not revert upon cooling

592 
595

596

FIG. $1\left(\mathrm{CoFe}_{2}\right)_{1 / 3} \mathrm{C}_{2} \mathrm{O}_{4}$ particles synthesized with different amount of PVA: (a) 0.0, (b)

597 1.0 , (c) 3.0 and (d) $5.0 \% \mathrm{w} / \mathrm{w}$.

598

FIG. $2 \mathrm{CoFe}_{2} \mathrm{O}_{4}$ particles synthesized with different amounts of PVA after calcination.

600

(a) $0.0 \%$, (b) $1.0 \%$, (c) $2.0 \%$, (d) $3.0 \%$, (e) $4.0 \%$ and (f) $5.0 \% \mathrm{w} / \mathrm{w}$.

601

602

FIG. 3. (a) Length and section of the synthesized $\mathrm{CoFe}_{2} \mathrm{O}_{4}$ nanobars as a function of

603

PVA concentration. (b) Molar ratio of metal cations to alcohol hydroxyl groups as a

604

function of PVA concentration in the precursor synthesis.

605

606

FIG. 4. SEM images of (a) precursor oxalate particles; (b) final ferrite particles.

607

FIG. 5. XRD Patterns of cobalt ferrite and oxalate particles for different PVA synthesis

609 concentrations.

610

611

FIG. 6. (a) magnetization curves for different PVA contents in the synthesis; (b)

612 Normalized magnetization curves. Insets: expanded view of the low field region. 
614 FIG. 7. Thermogravimetric curves measured for $\mathrm{CoFe}_{2} \mathrm{O}_{4}$, PANI and $\mathrm{CoFe}_{2} \mathrm{O}_{4}-\mathrm{PANI}$ 615 composites.

616

617 FIG. 8. Typical SEM images of $\mathrm{CoFe}_{2} \mathrm{O}_{4}$ nanorods-PANI of different feed 618 compositions: (a) and (b), $r_{S}=6$; (c) and (d), $r_{S}=12 ;$ (e) $r_{S}=20 ;(\mathrm{f}),(\mathrm{g})$ and (h), $r_{S}=16$. 619 In image pairs (a) - (b), (c) - d), and (g) - (h) the same region of the sample was imaged 620 with two different detectors: InLens in and QBSD, respectively. The same particle is 621 encircled in each case for better comparison.

622

623 FIG. 9. IR spectra for the $1800-400 \mathrm{~cm}^{-1}$ region of samples of: a) $\mathrm{CoFe}_{2} \mathrm{O}_{4} 3.0 \%$ PVA, 624 b) pure PANI, and $\mathrm{CoFe}_{2} \mathrm{O}_{4}$-PANI composites c) $r_{S}=6$ and d) $r_{S}=16$.

625

626 FIG. 10. Magnetization curves for $\mathrm{CoFe}_{2} \mathrm{O}_{4}$-PANI composites for different $r_{S}$ values: (a)

627 relative to the total mass of the composite (b) relative to the mass of cobalt ferrite within 628 the composite sample.

629 


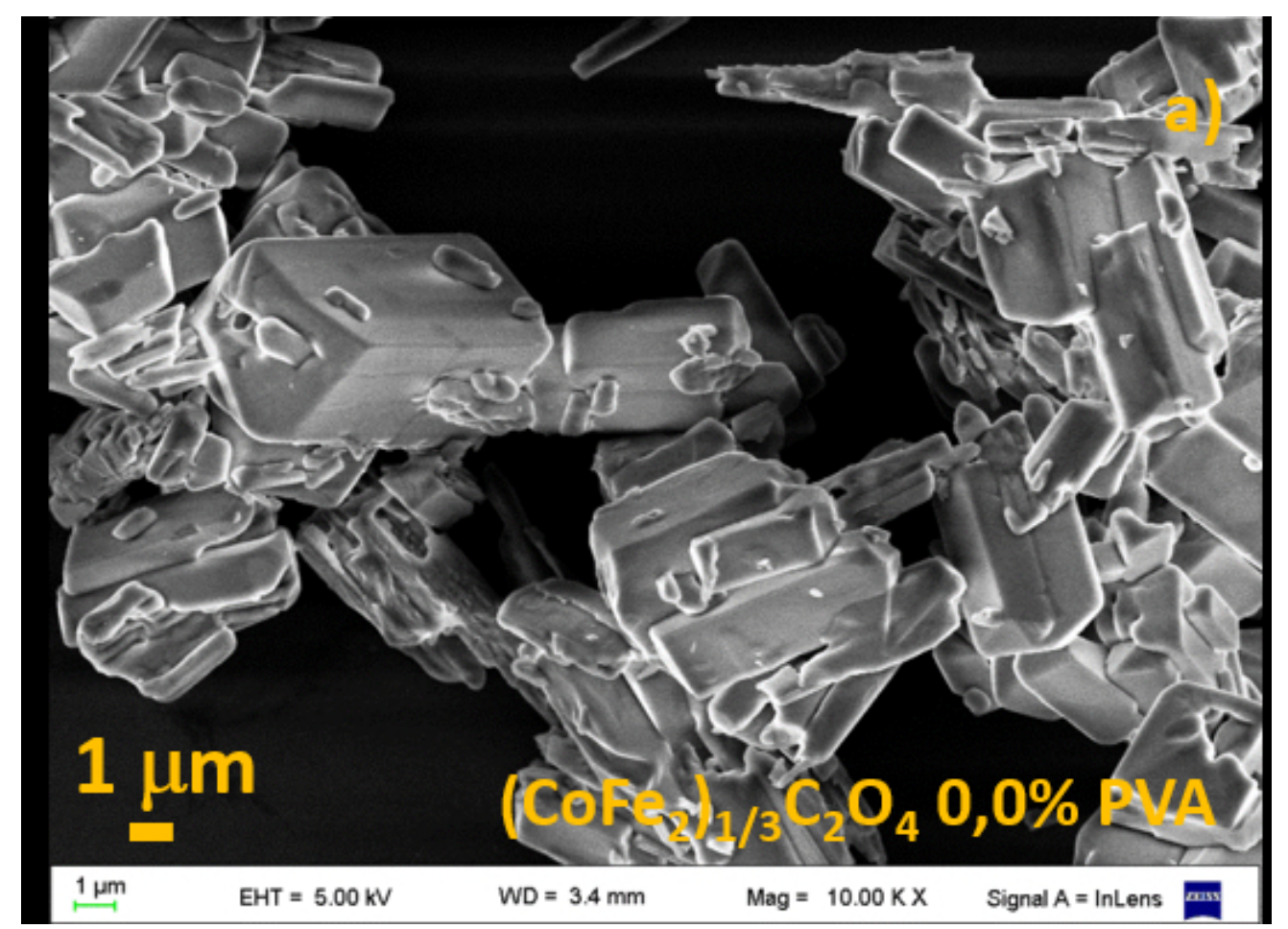

FIG. 1 (CoFe2)1/3C204 particles synthesized with different amount of PVA: (a) 0.0, (b) 1.0, (c) 3.0 and (d) $5.0 \% \mathrm{w} / \mathrm{w}$.

$91 \times 66 \mathrm{~mm}(150 \times 150$ DPI $)$ 


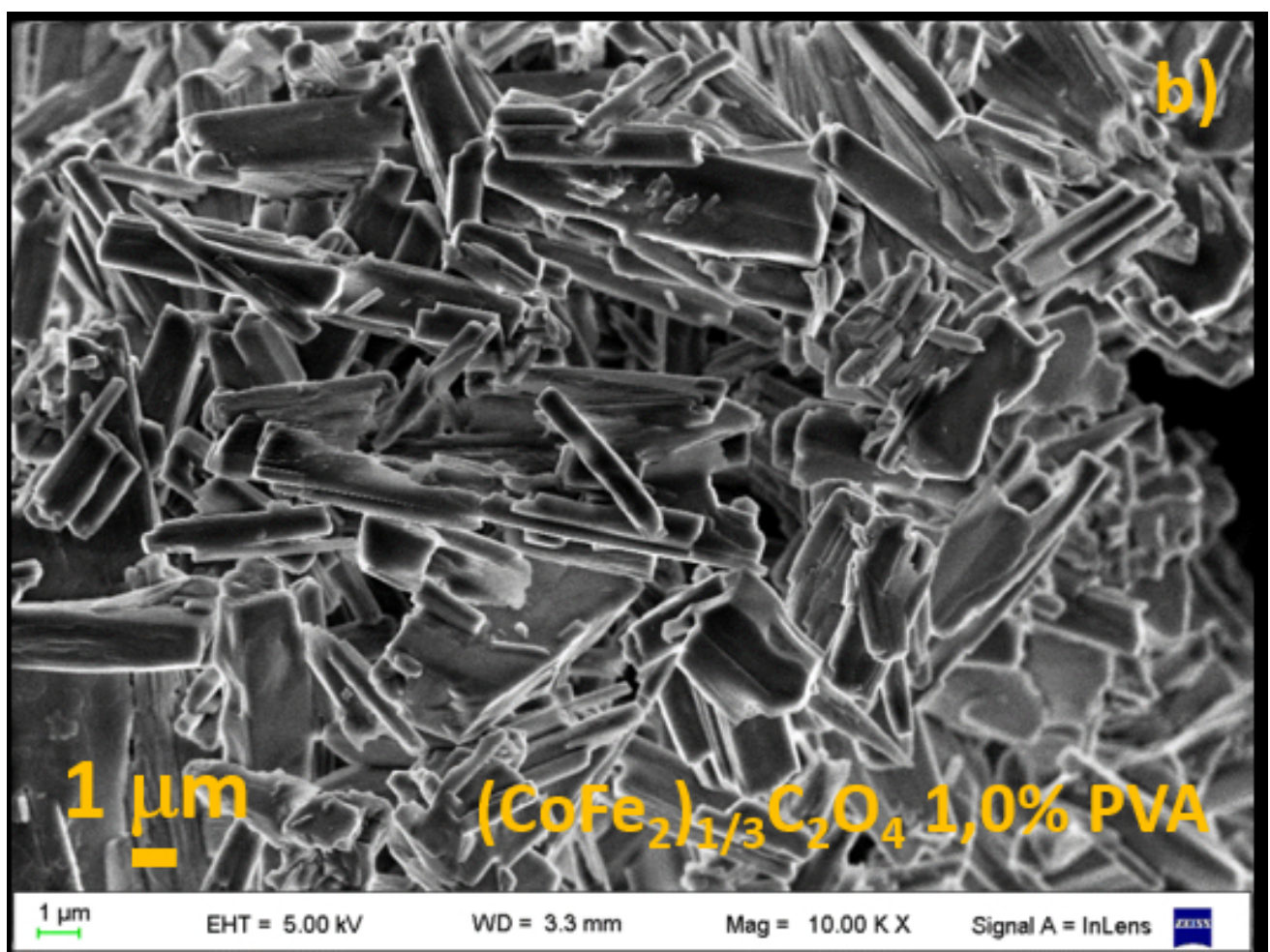

FIG. 1 (CoFe2)1/3C2O4 particles synthesized with different amount of PVA: (a) 0.0, (b) 1.0, (c) 3.0 and (d) $5.0 \% \mathrm{w} / \mathrm{w}$.

$90 \times 67 \mathrm{~mm}(150 \times 150 \mathrm{DPI})$ 


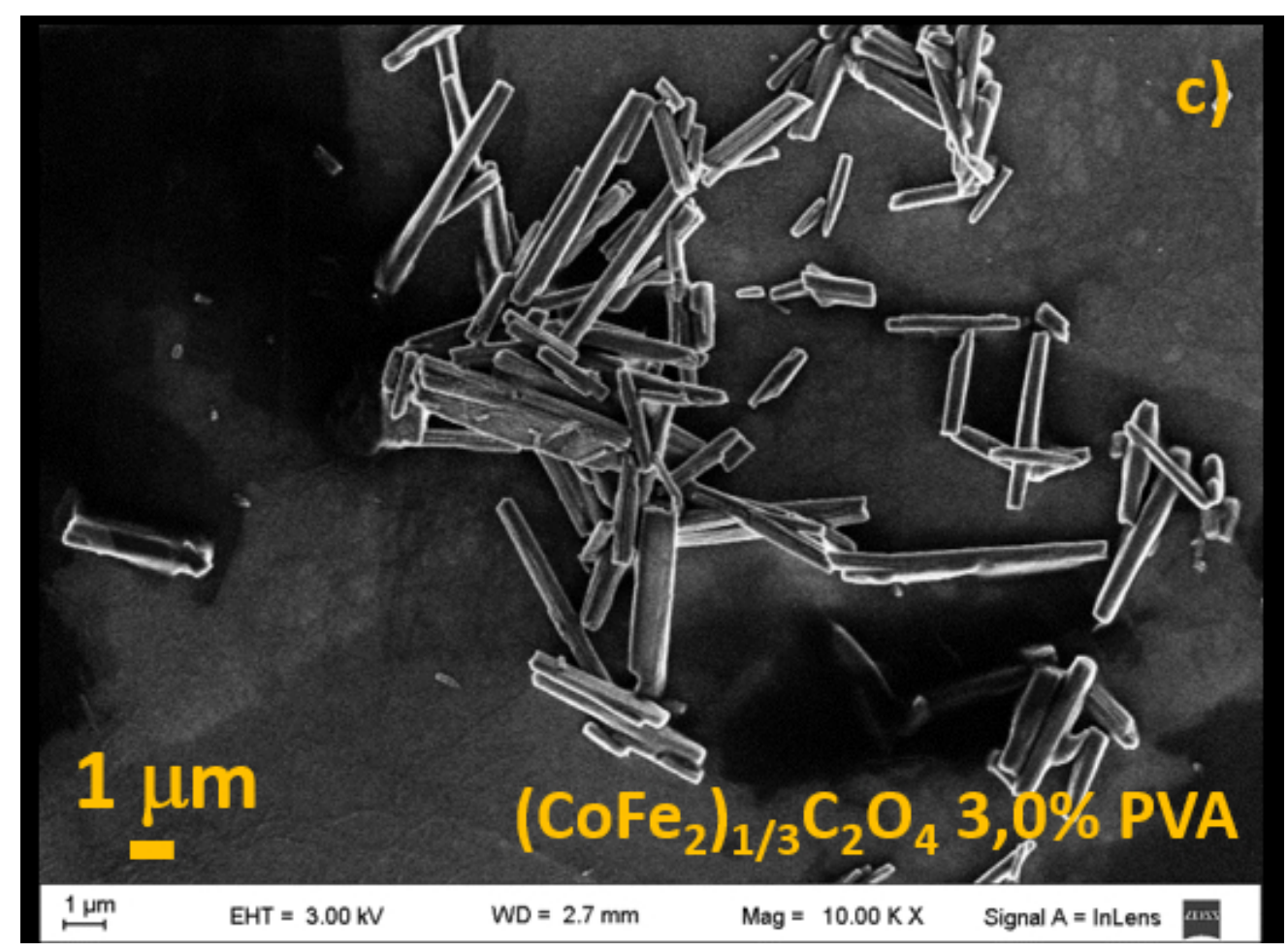

FIG. 1 (CoFe2)1/3C2O4 particles synthesized with different amount of PVA: (a) 0.0, (b) 1.0, (c) 3.0 and (d) $5.0 \% \mathrm{w} / \mathrm{w}$.

$91 \times 67 \mathrm{~mm}(150 \times 150 \mathrm{DPI})$ 


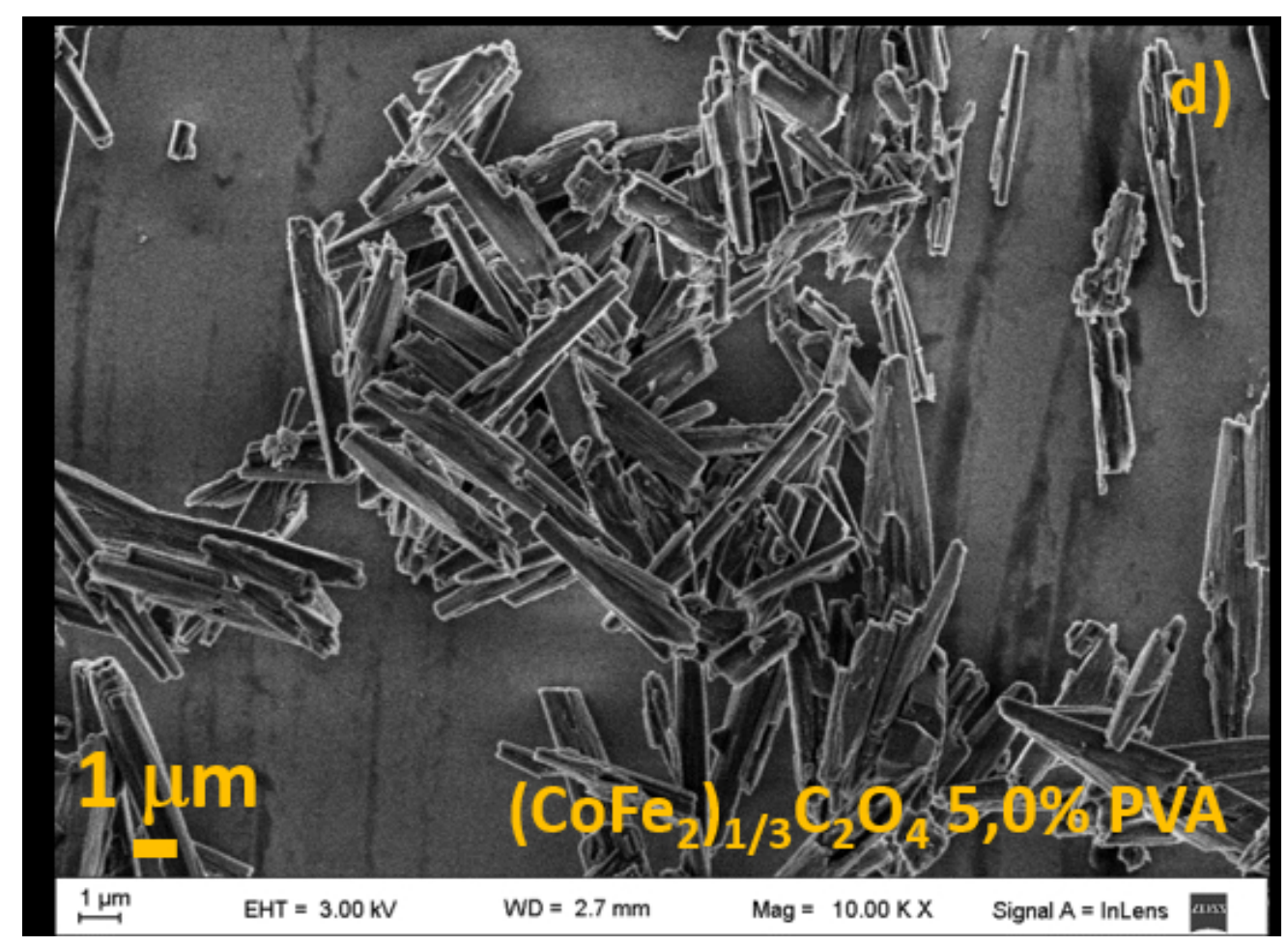

FIG. 1 (CoFe2)1/3C2O4 particles synthesized with different amount of PVA: (a) 0.0, (b) 1.0, (c) 3.0 and (d) $5.0 \% \mathrm{w} / \mathrm{w}$.

$92 \times 67 \mathrm{~mm}(150 \times 150 \mathrm{DPI})$ 


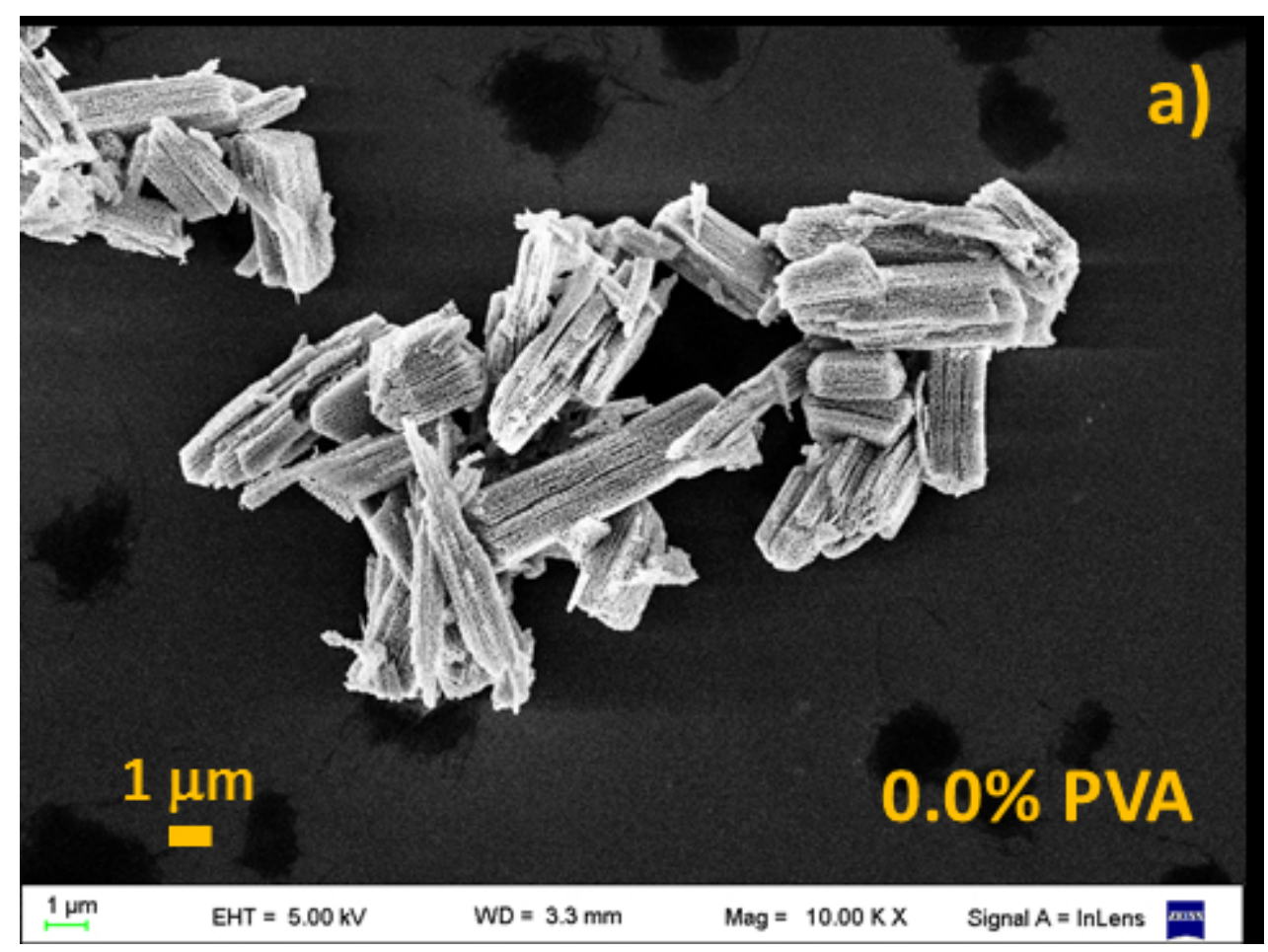

FIG. 2 CoFe2O4 particles synthesized with different amounts of PVA after calcination. (a) $0.0 \%$, (b) $1.0 \%$, (c) $2.0 \%$, (d) $3.0 \%$, (e) $4.0 \%$ and (f) $5.0 \% \mathrm{w} / \mathrm{w}$.

$81 \times 61 \mathrm{~mm}(150 \times 150 \mathrm{DPI})$ 


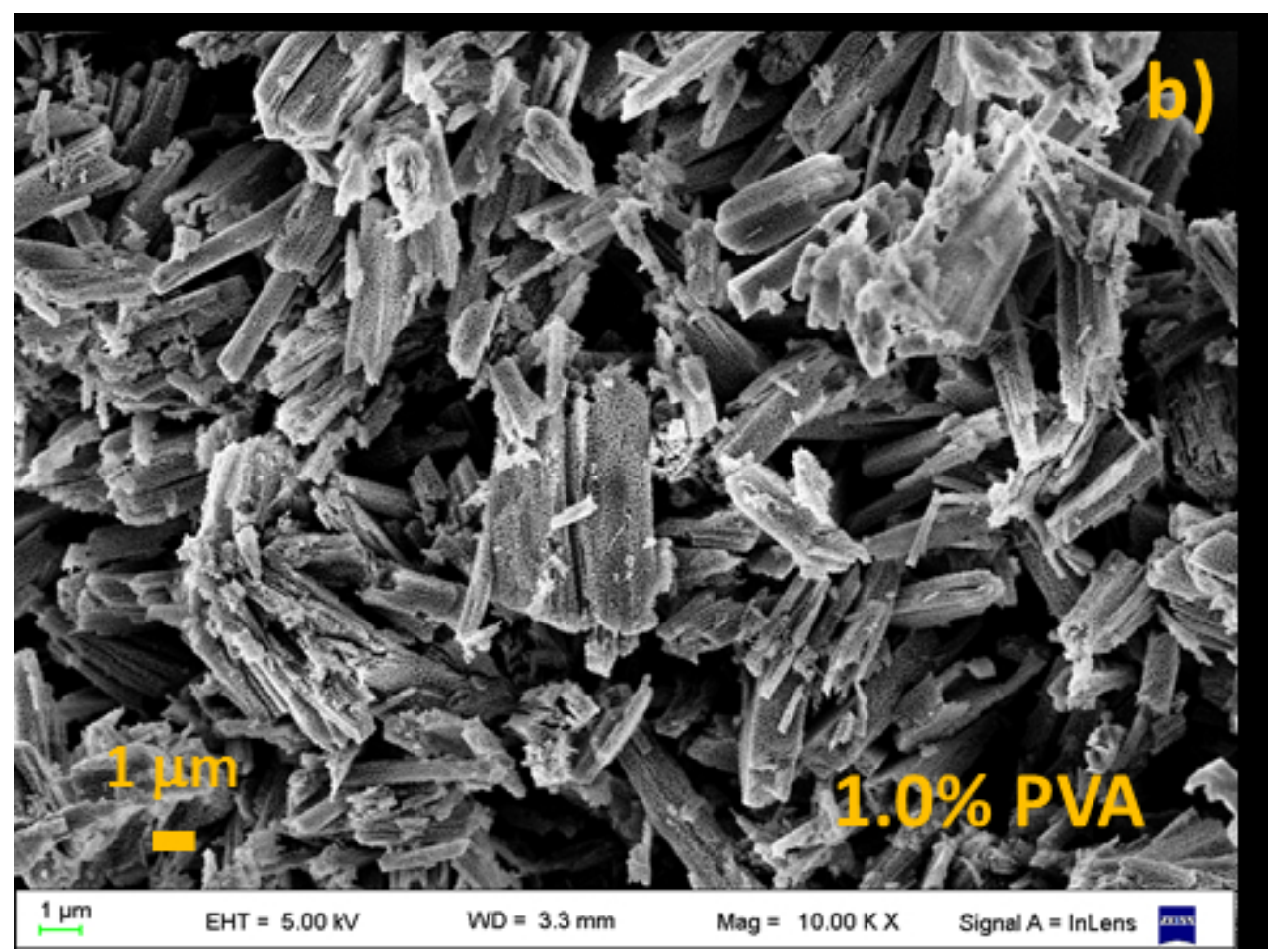

FIG. 2 CoFe2O4 particles synthesized with different amounts of PVA after calcination. (a) $0.0 \%$, (b) $1.0 \%$, (c) $2.0 \%$, (d) $3.0 \%$, (e) $4.0 \%$ and (f) $5.0 \% \mathrm{w} / \mathrm{w}$.

$82 \times 61 \mathrm{~mm}(150 \times 150 \mathrm{DPI})$ 


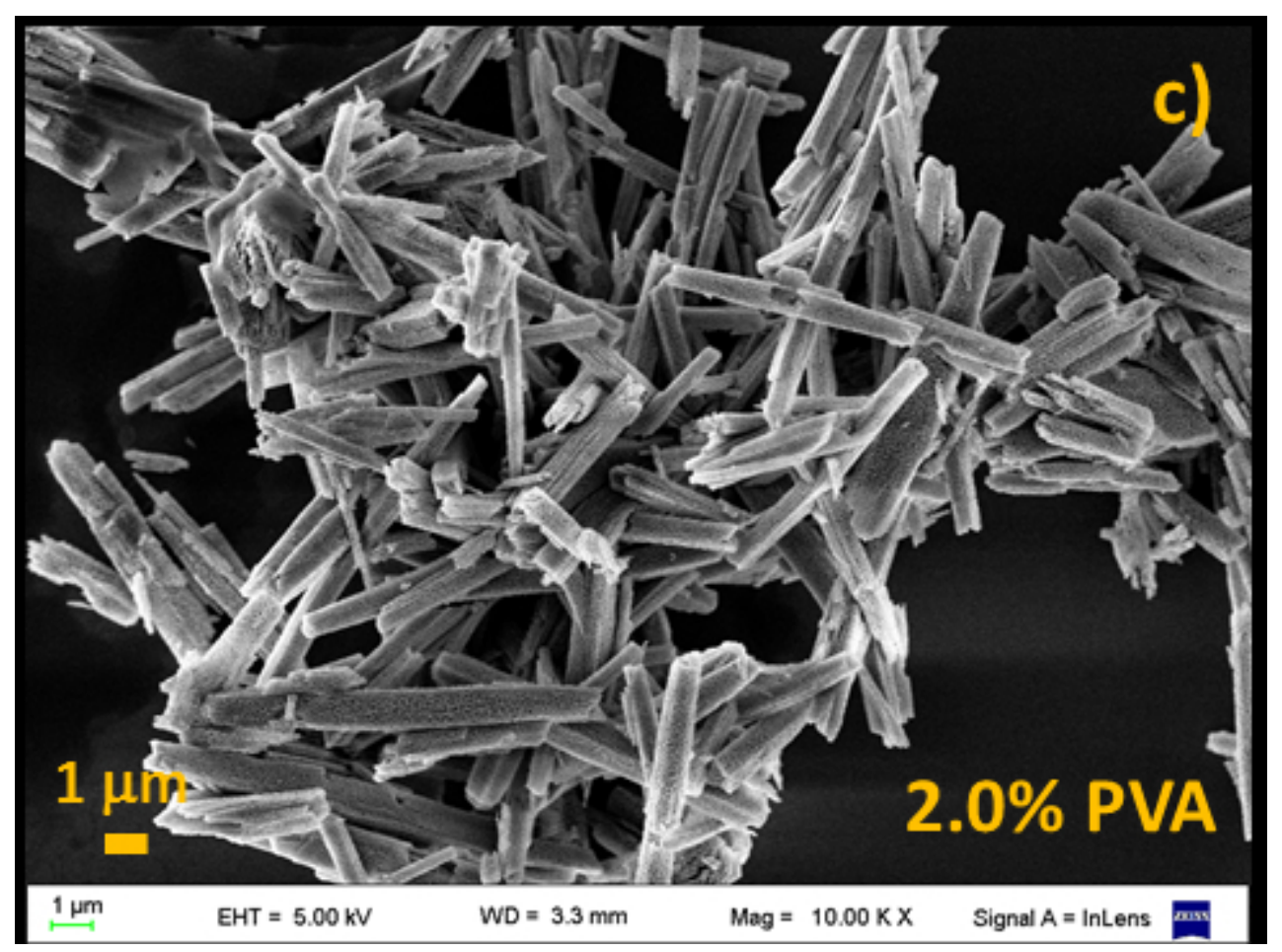

FIG. 2 CoFe2O4 particles synthesized with different amounts of PVA after calcination. (a) $0.0 \%$, (b) $1.0 \%$, (c) $2.0 \%$, (d) $3.0 \%$, (e) $4.0 \%$ and (f) $5.0 \% \mathrm{w} / \mathrm{w}$.

$82 \times 61 \mathrm{~mm}(150 \times 150 \mathrm{DPI})$ 


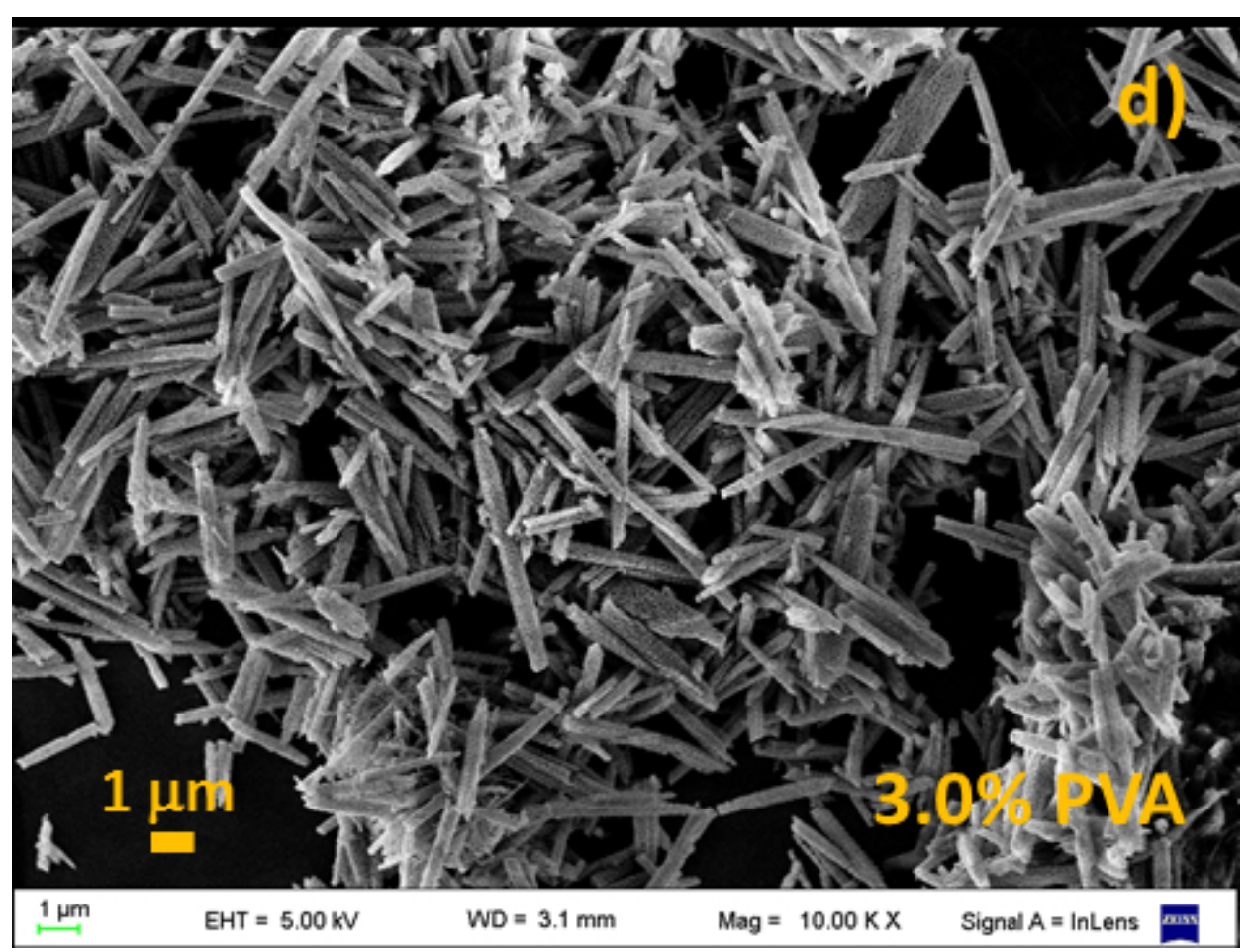

FIG. 2 CoFe2O4 particles synthesized with different amounts of PVA after calcination. (a) $0.0 \%$, (b) $1.0 \%$, (c) $2.0 \%$, (d) $3.0 \%$, (e) $4.0 \%$ and (f) $5.0 \% \mathrm{w} / \mathrm{w}$.

$80 \times 61 \mathrm{~mm}(150 \times 150 \mathrm{DPI})$ 


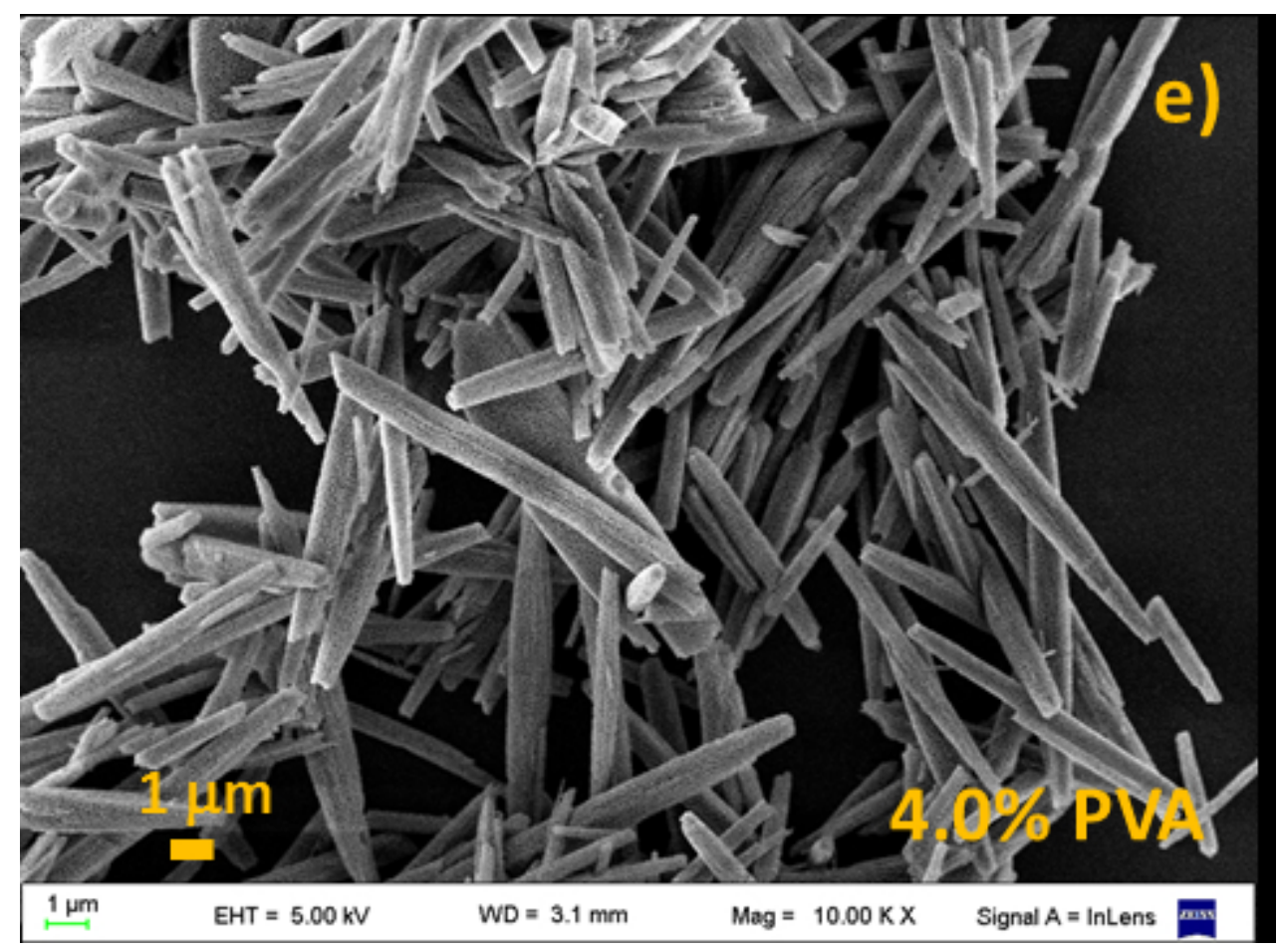

FIG. 2 CoFe2O4 particles synthesized with different amounts of PVA after calcination. (a) $0.0 \%$, (b) $1.0 \%$, (c) $2.0 \%$, (d) $3.0 \%$, (e) $4.0 \%$ and (f) $5.0 \% \mathrm{w} / \mathrm{w}$.

$81 \times 60 \mathrm{~mm}(150 \times 150 \mathrm{DPI})$ 


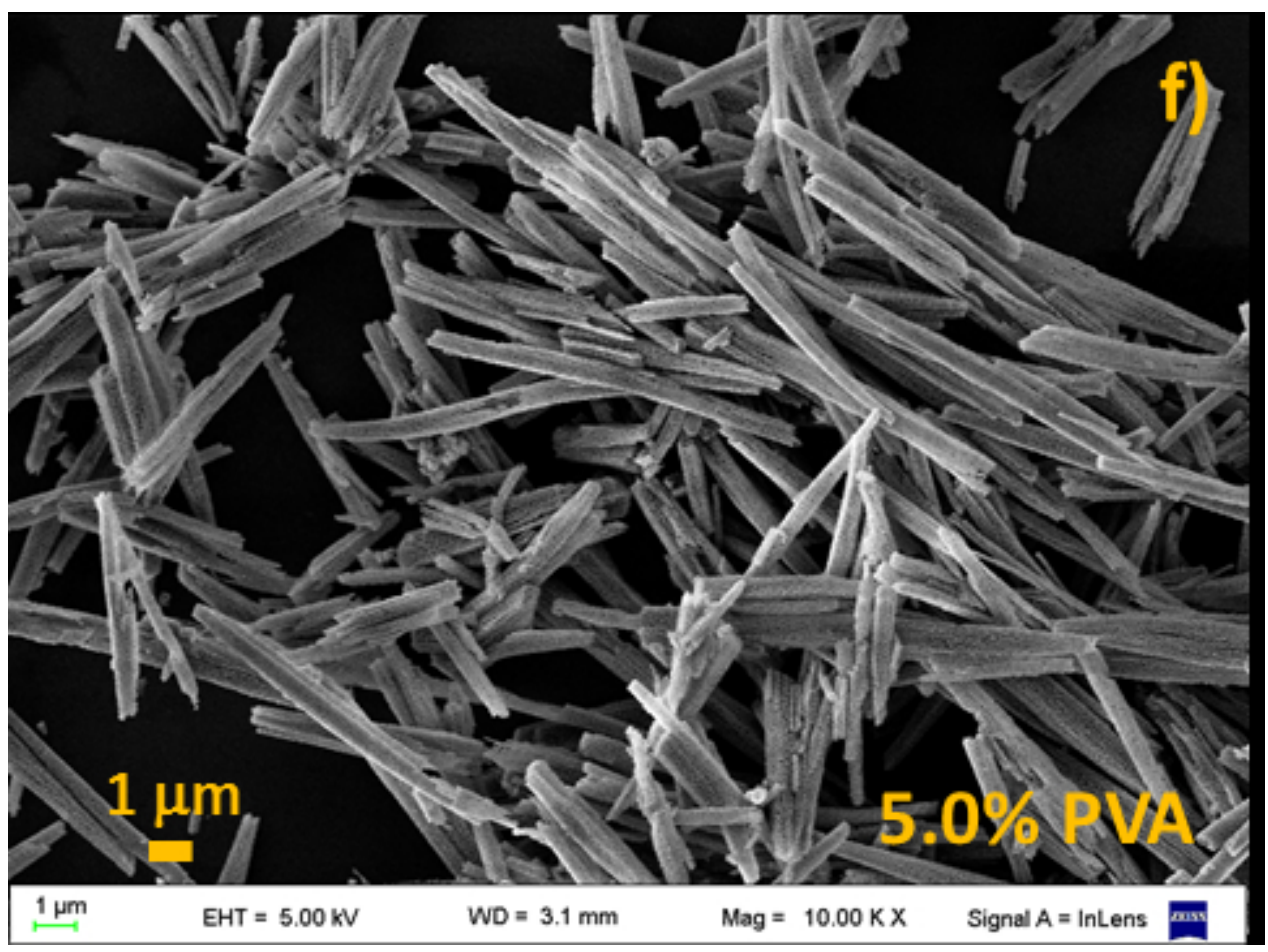

FIG. 2 CoFe2O4 particles synthesized with different amounts of PVA after calcination. (a) $0.0 \%$, (b) $1.0 \%$, (c) $2.0 \%$, (d) $3.0 \%$, (e) $4.0 \%$ and (f) $5.0 \% \mathrm{w} / \mathrm{w}$.

$81 \times 60 \mathrm{~mm}(150 \times 150$ DPI $)$ 


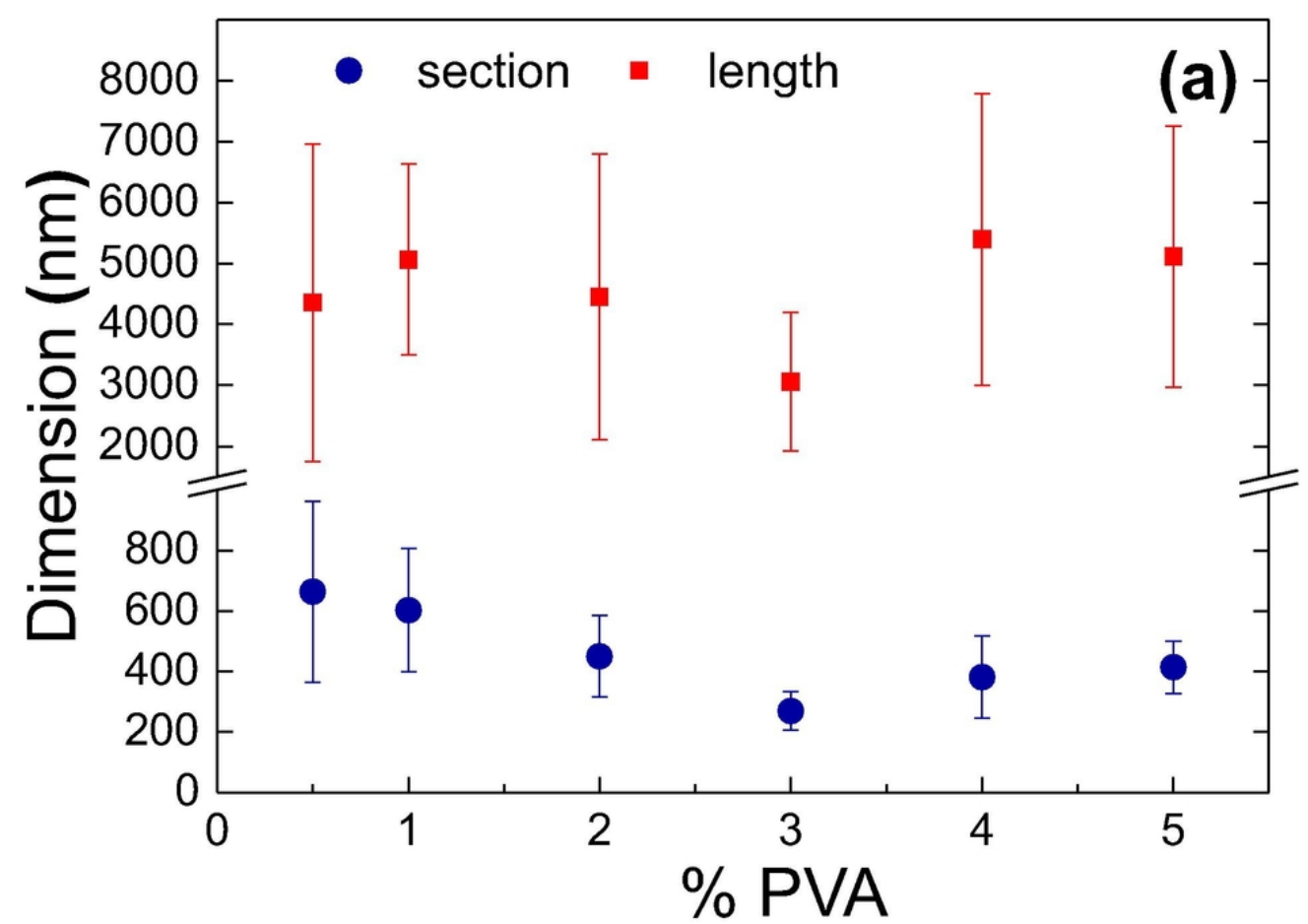

FIG. 3. (a) Length and section of the synthesized CoFe2O4 nanobars as a function of PVA concentration. (b) Molar ratio of metal cations to alcohol hydroxyl groups as a function of PVA concentration in the precursor synthesis.

$81 \times 57 \mathrm{~mm}(300 \times 300 \mathrm{DPI})$ 


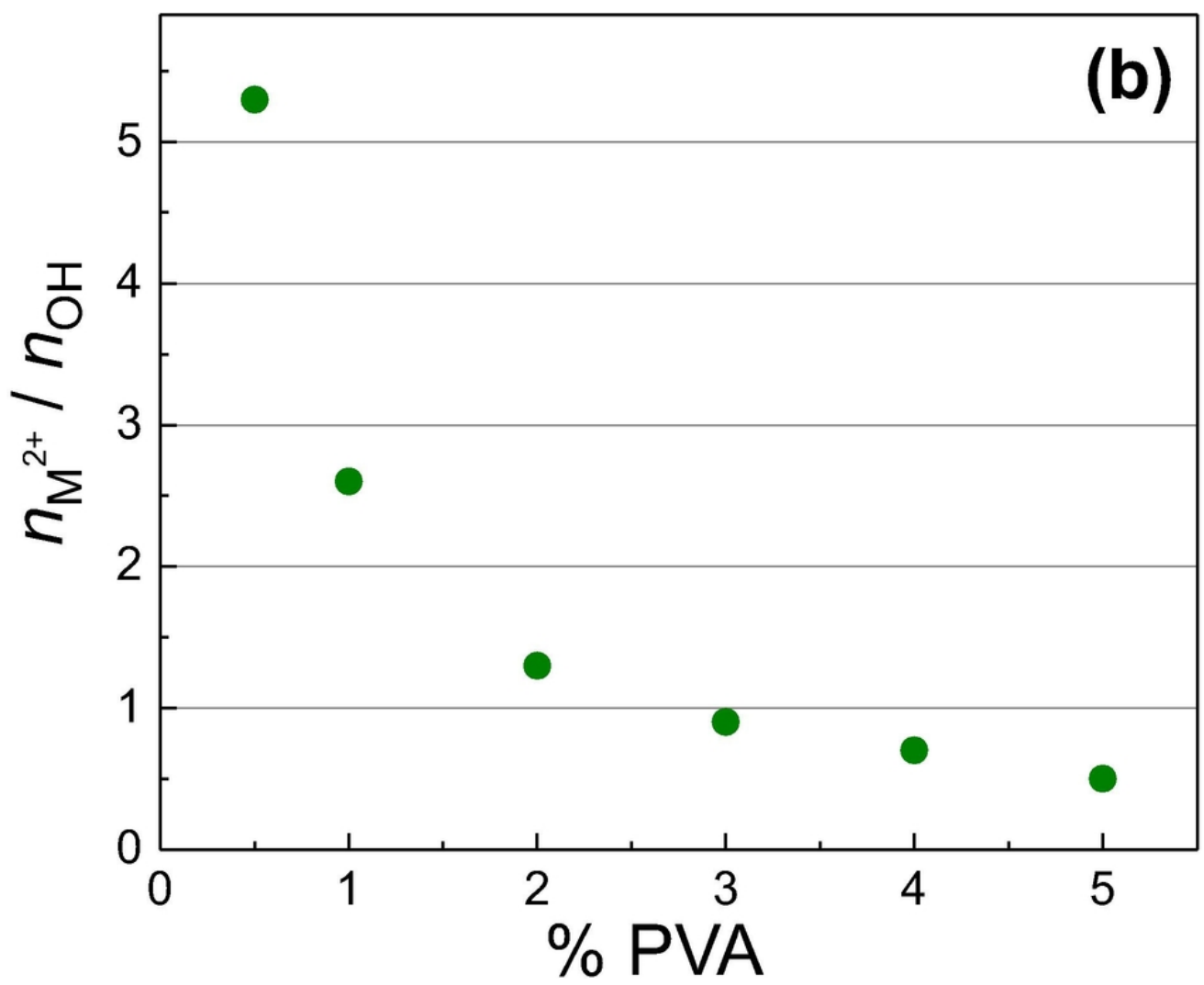

FIG. 3. (a) Length and section of the synthesized CoFe2O4 nanobars as a function of PVA concentration. (b) Molar ratio of metal cations to alcohol hydroxyl groups as a function of PVA concentration in the precursor synthesis.

$80 \times 65 \mathrm{~mm}(300 \times 300 \mathrm{DPI})$ 


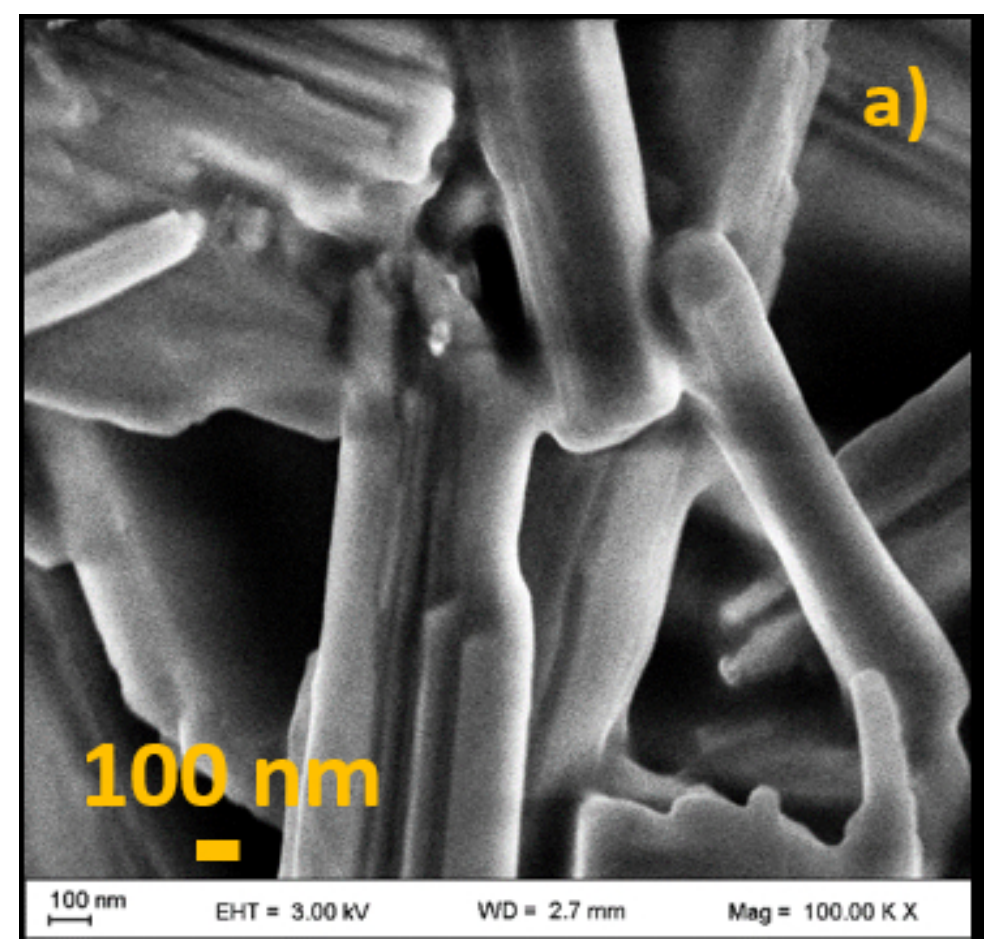

FIG. 4. SEM images of (a) precursor oxalate particles; (b) final ferrite particles. $62 \times 60 \mathrm{~mm}(150 \times 150 \mathrm{DPI})$ 


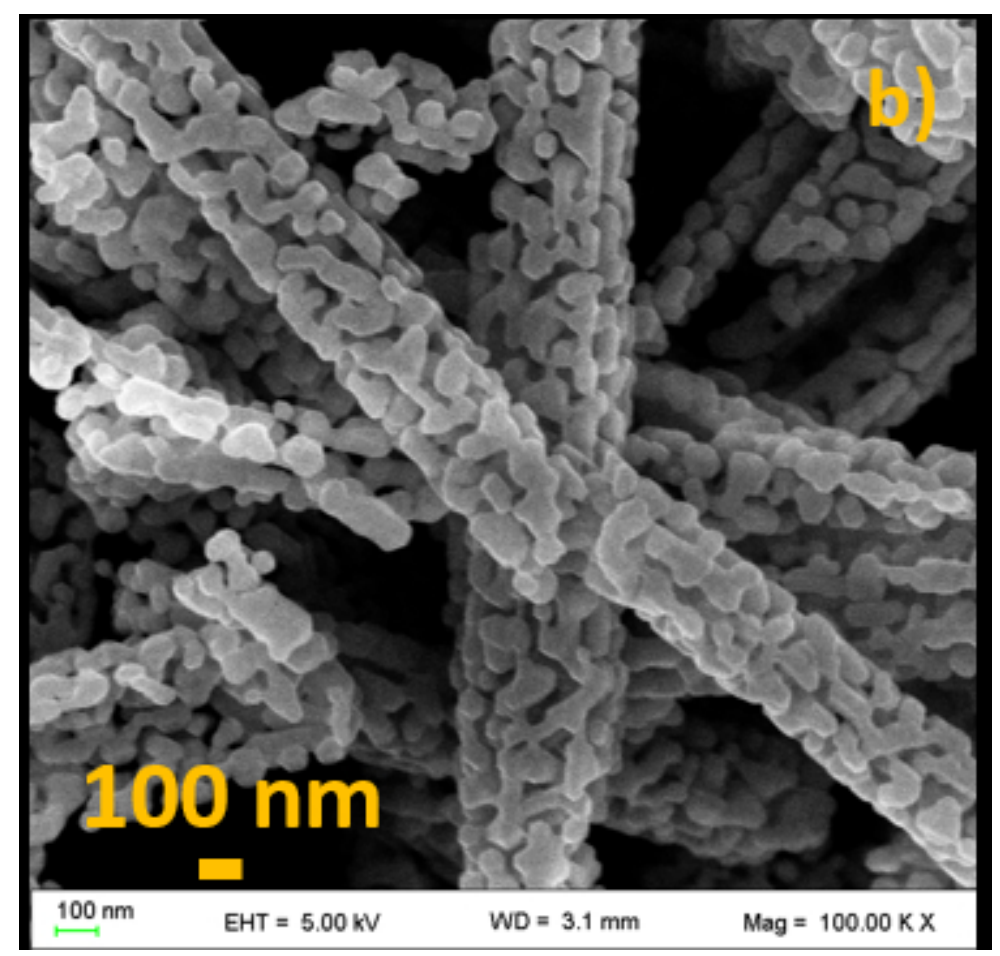

FIG. 4. SEM images of (a) precursor oxalate particles; (b) final ferrite particles. $63 \times 60 \mathrm{~mm}(150 \times 150 \mathrm{DPI})$ 


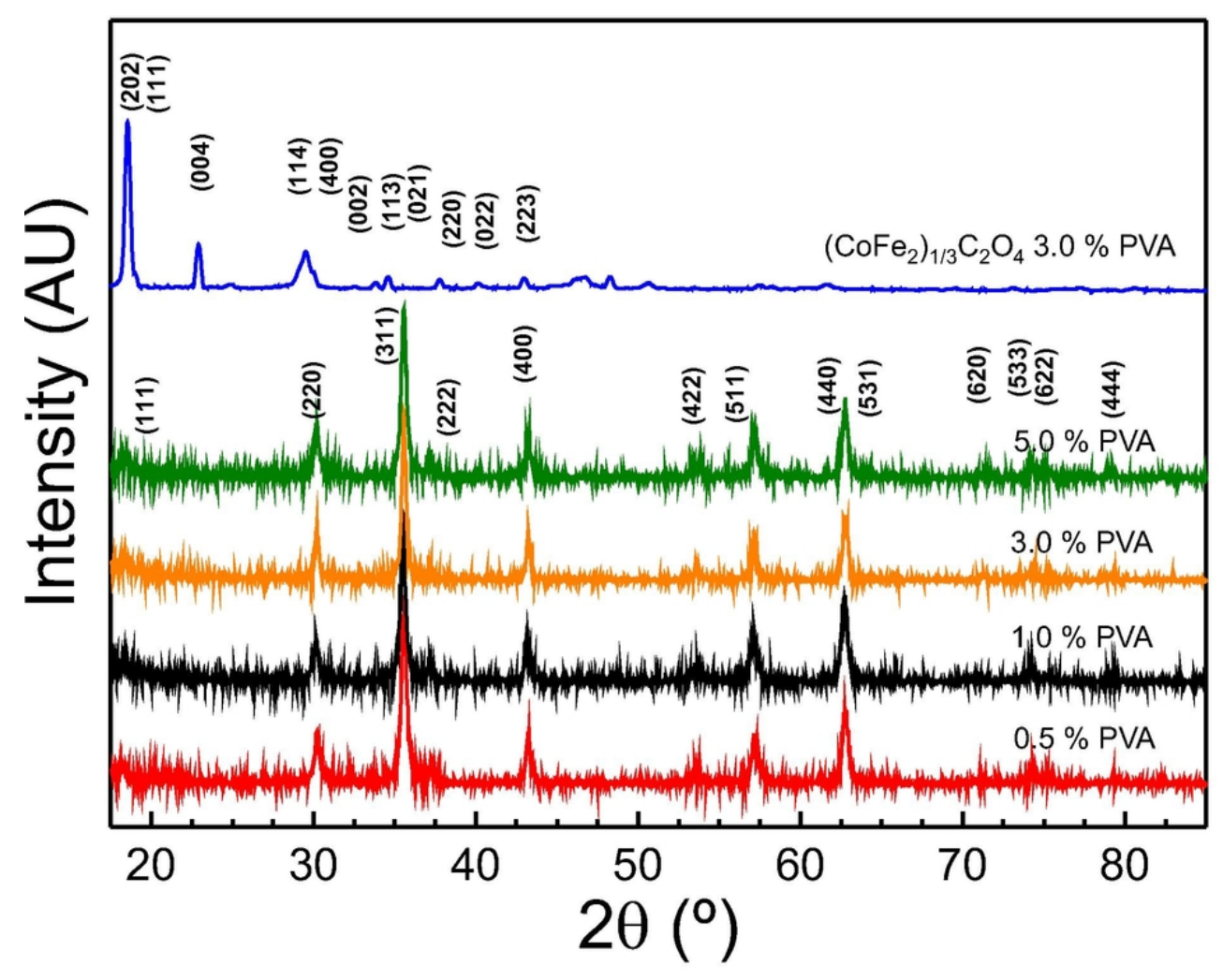

FIG. 5. XRD Patterns of cobalt ferrite and oxalate particles for different PVA synthesis concentrations.

$81 \times 65 \mathrm{~mm}(300 \times 300$ DPI) 


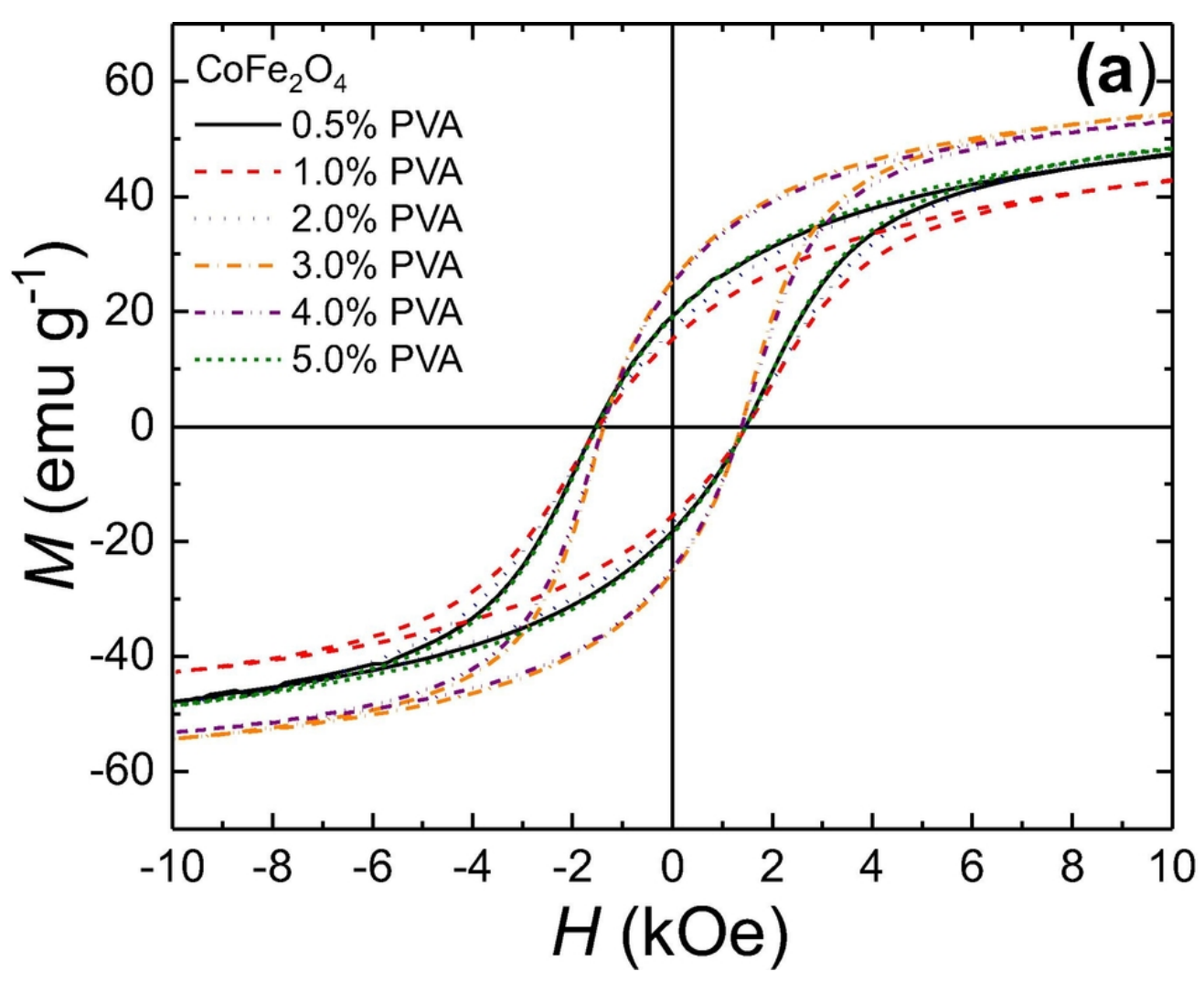

FIG. 6. (a) magnetization curves for different PVA contents in the synthesis; (b) Normalized magnetization curves. Insets: expanded view of the low field region.

$81 \times 64 \mathrm{~mm}(300 \times 300$ DPI) 


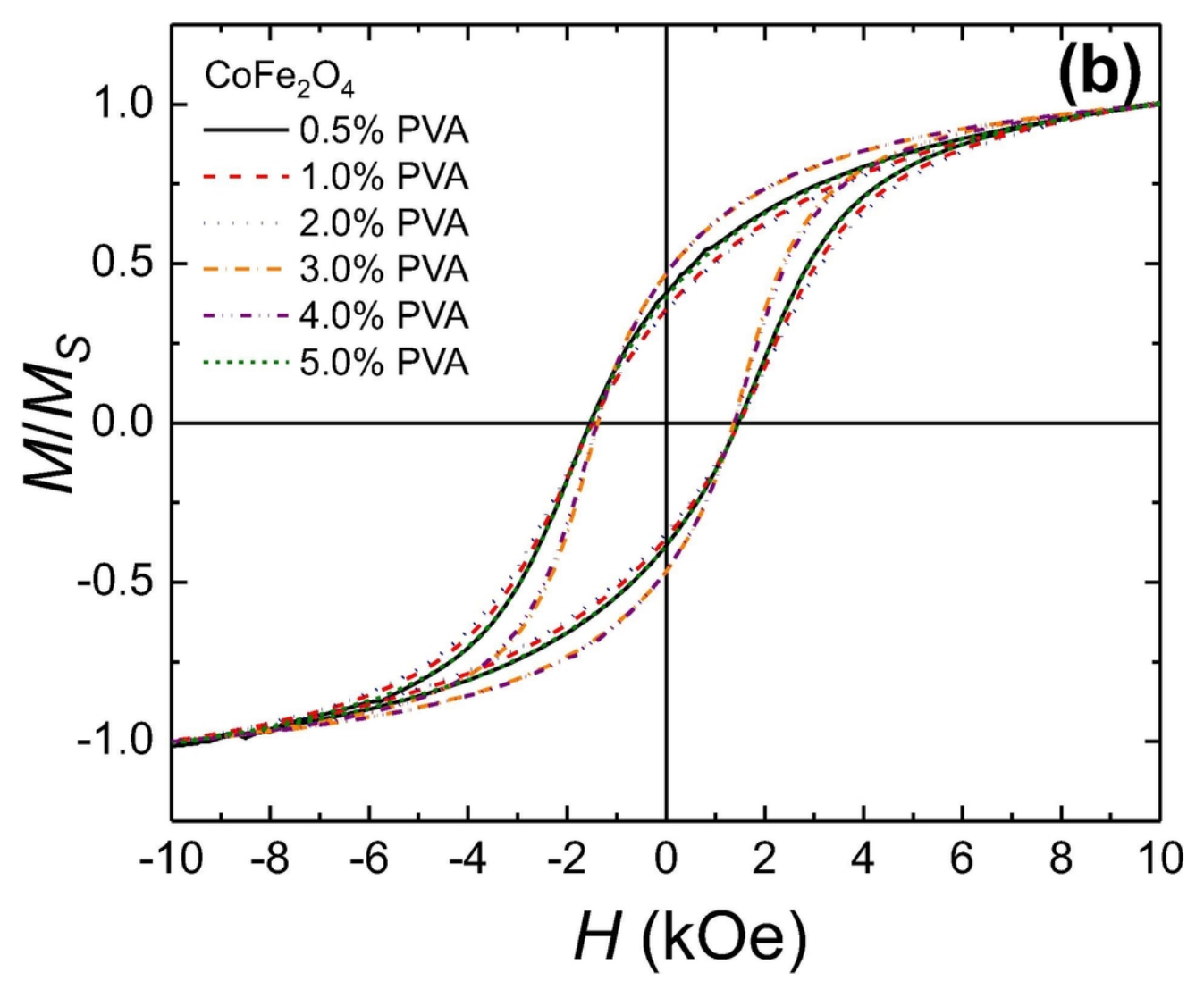

FIG. 6. (a) magnetization curves for different PVA contents in the synthesis; (b) Normalized magnetization curves. Insets: expanded view of the low field region.

$81 \times 66 \mathrm{~mm}(300 \times 300 \mathrm{DPI})$ 


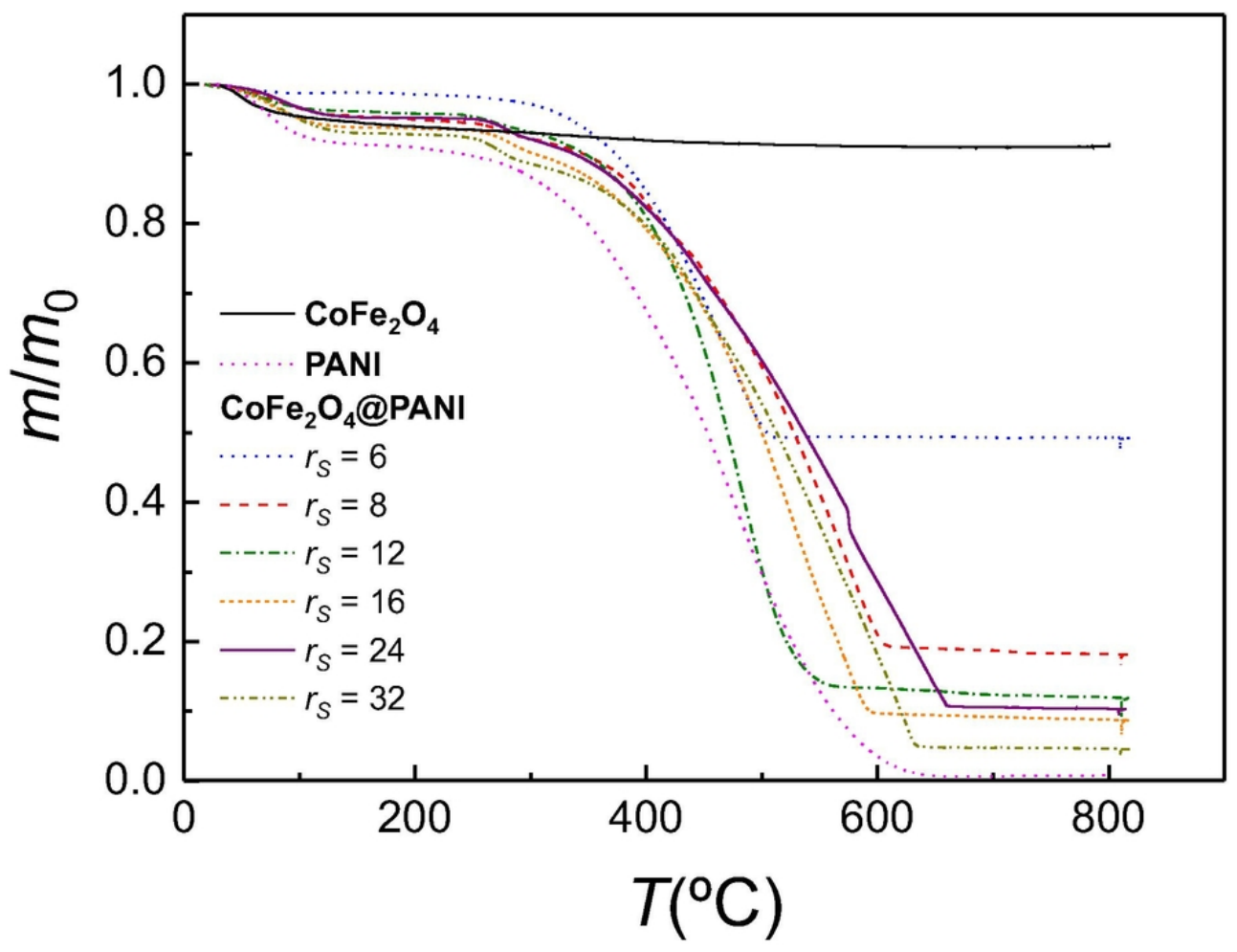

FIG. 7. Thermogravimetric curves measured for CoFe2O4, PANI and CoFe2O4-PANI composites. $81 \times 63 \mathrm{~mm}(300 \times 300 \mathrm{DPI})$ 


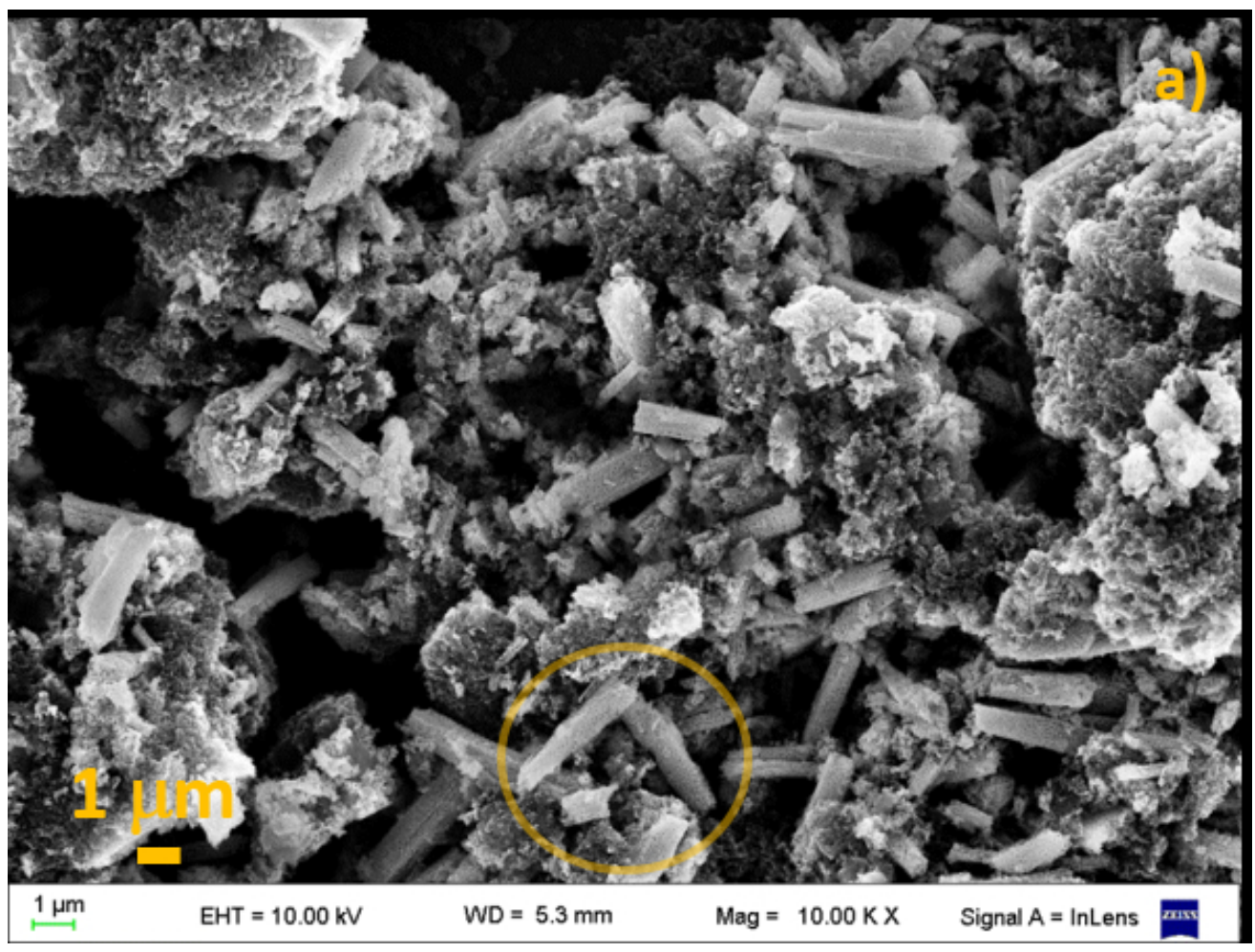

FIG. 8. Typical SEM images of CoFe2O4 nanorods-PANI of different feed compositions: (a) and (b), rS = 6; (c) and (d), rS = 12; (e) rS = 20; (f), (g) and (h), rS = 16. In image pairs (a) - (b), (c) - d), and (g) - (h) the same region of the sample was imaged with two different detectors: InLens in and QBSD, respectively. The same particle is encircled in each case for better comparison.

$100 \times 75 \mathrm{~mm}(150 \times 150 \mathrm{DPI})$ 


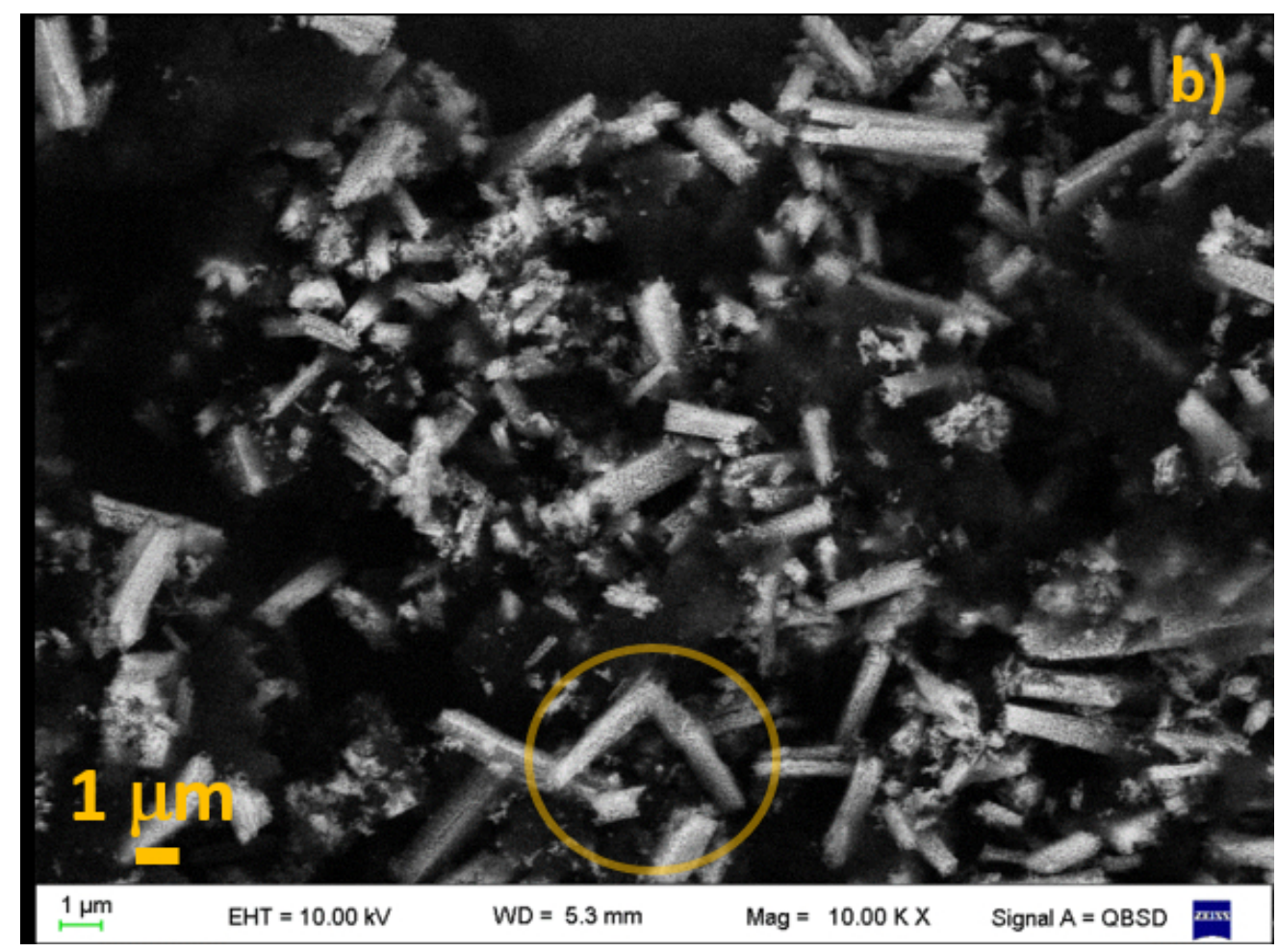

FIG. 8. Typical SEM images of CoFe2O4 nanorods-PANI of different feed compositions: (a) and (b), rS = 6; (c) and (d), rS = 12; (e) rS = 20; (f), (g) and (h), rS = 16. In image pairs (a) - (b), (c) - d), and (g) - (h) the same region of the sample was imaged with two different detectors: InLens in and QBSD, respectively. The same particle is encircled in each case for better comparison.

$101 \times 75 \mathrm{~mm}(150 \times 150 \mathrm{DPI})$ 


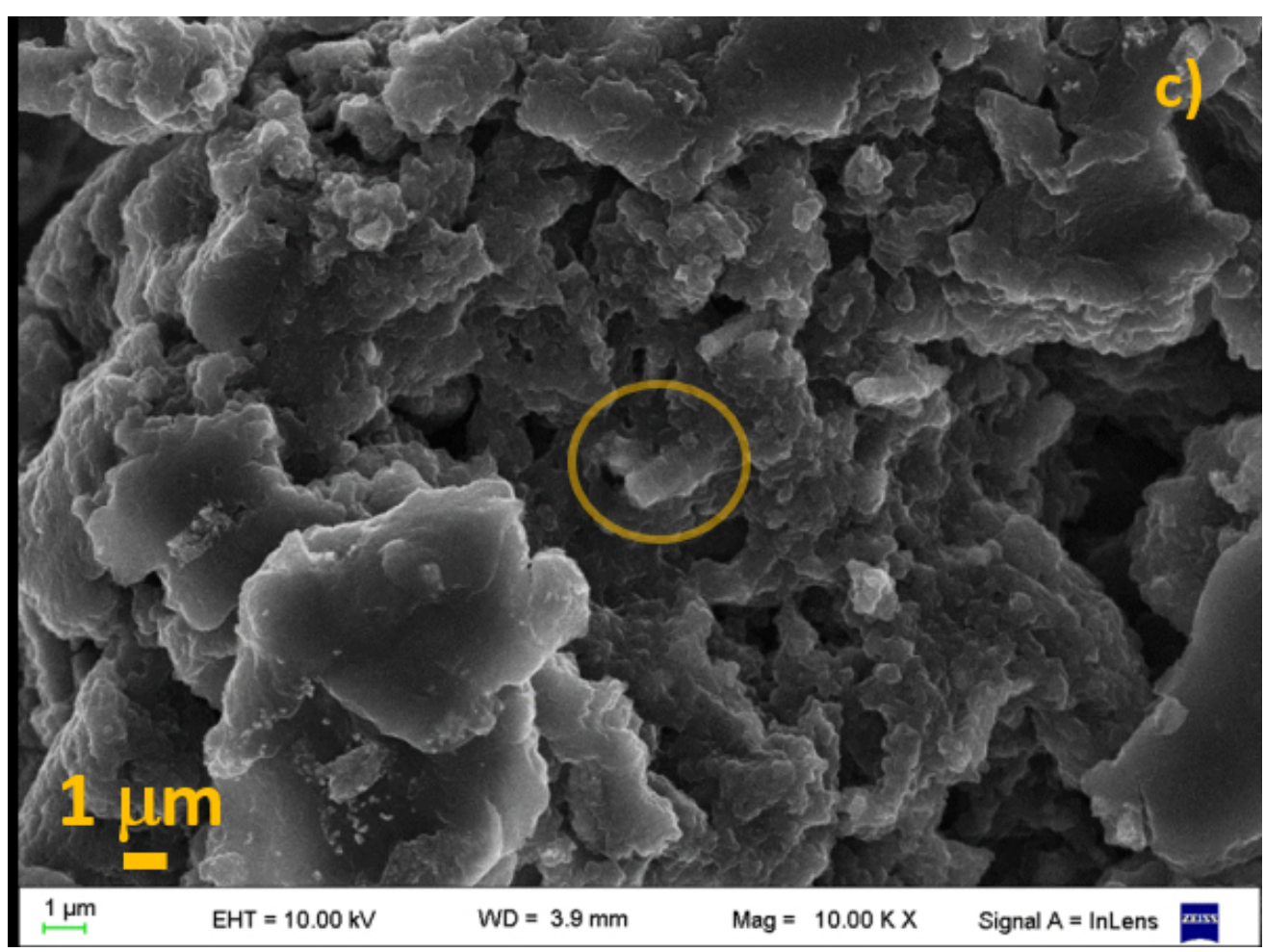

FIG. 8. Typical SEM images of CoFe2O4 nanorods-PANI of different feed compositions: (a) and (b), rS = 6; (c) and (d), rS = 12; (e) rS = 20; (f), (g) and (h), rS = 16. In image pairs (a) - (b), (c) - d), and (g) - (h) the same region of the sample was imaged with two different detectors: InLens in and QBSD, respectively. The same particle is encircled in each case for better comparison.

$100 \times 75 \mathrm{~mm}(150 \times 150 \mathrm{DPI})$ 


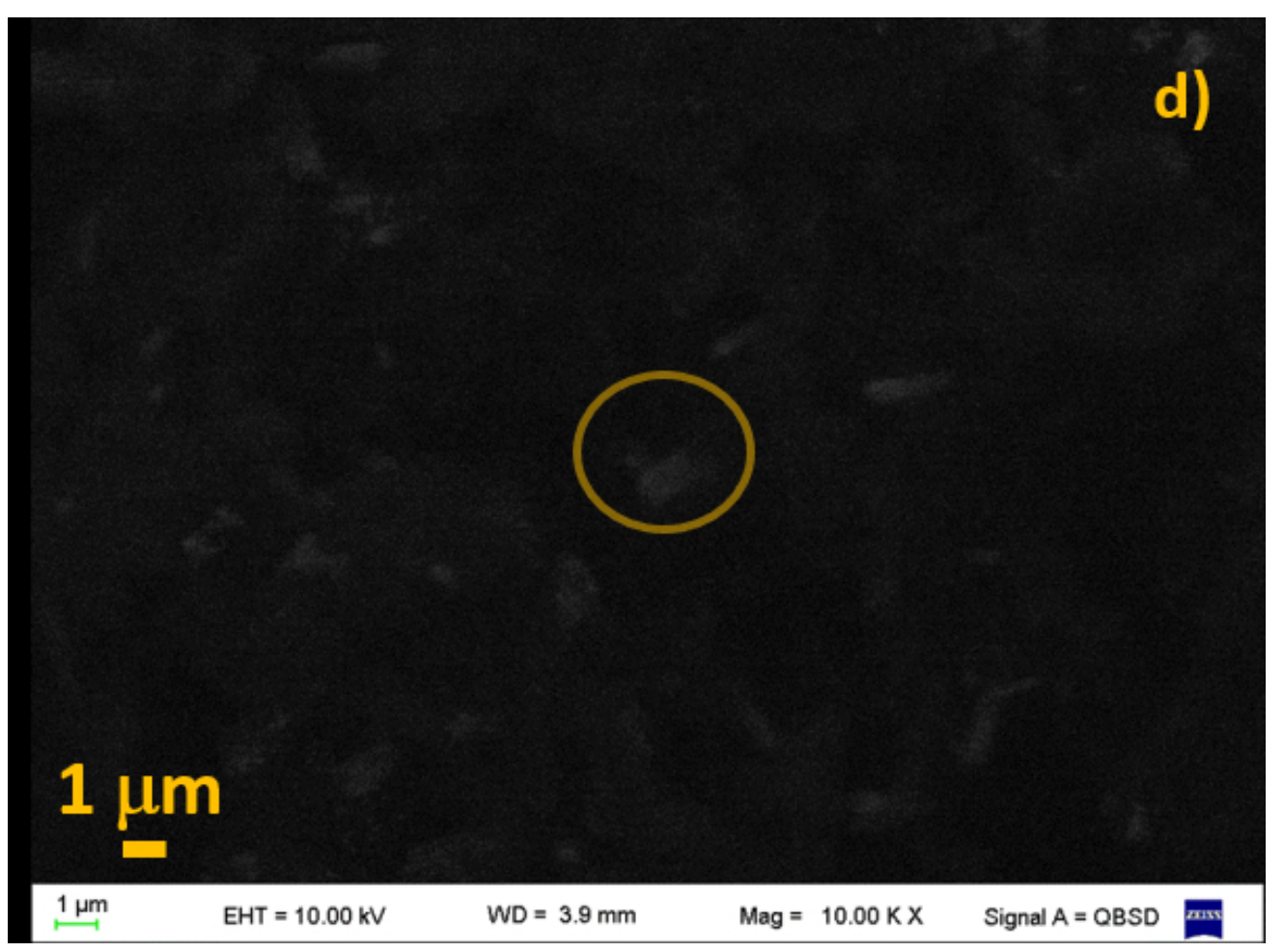

FIG. 8. Typical SEM images of CoFe2O4 nanorods-PANI of different feed compositions: (a) and (b), rS = 6; (c) and (d), rS = 12; (e) rS = 20; (f), (g) and (h), rS = 16. In image pairs (a) - (b), (c) - d), and (g) - (h) the same region of the sample was imaged with two different detectors: InLens in and QBSD, respectively. The same particle is encircled in each case for better comparison.

$101 \times 75 \mathrm{~mm}(150 \times 150 \mathrm{DPI})$ 


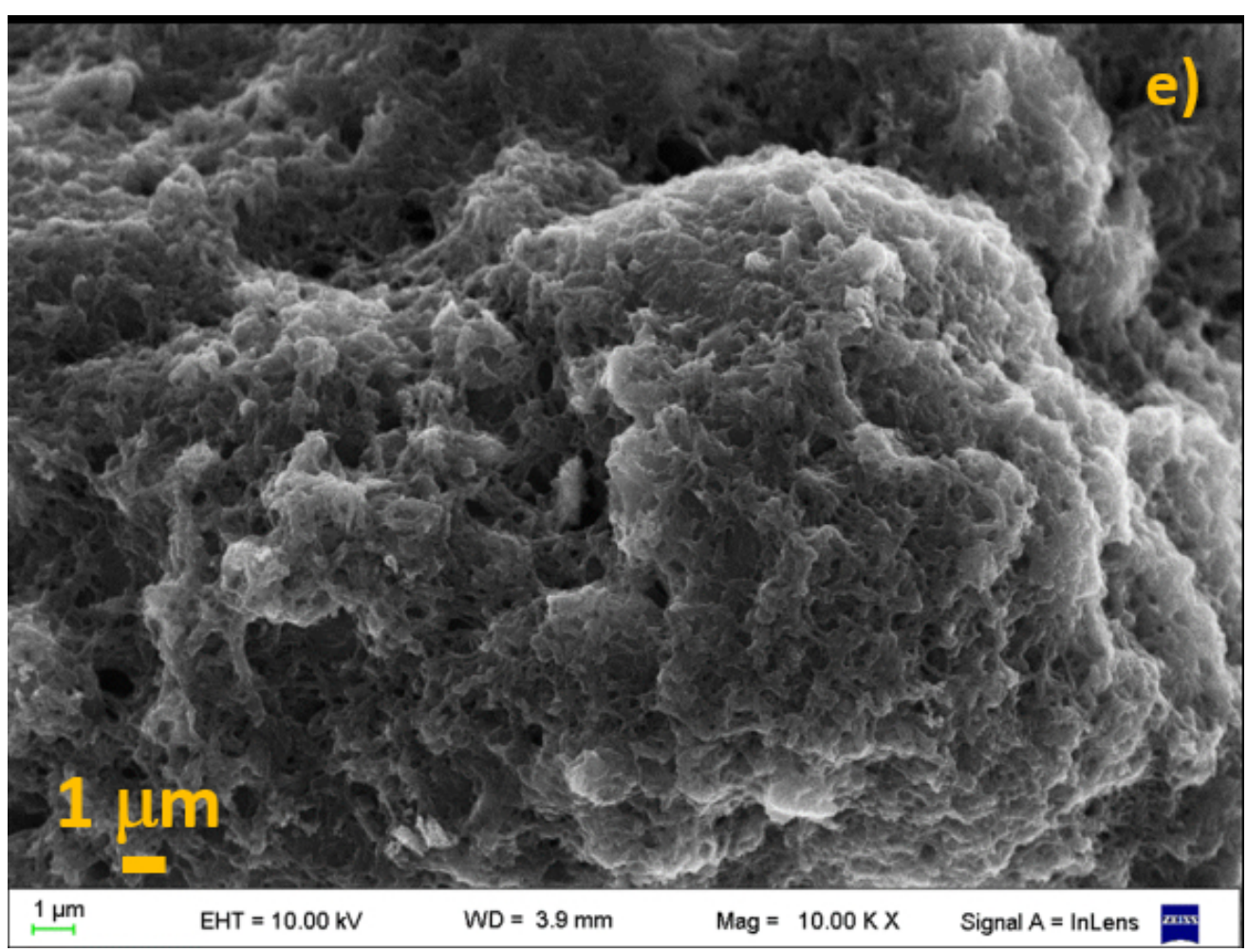

FIG. 8. Typical SEM images of CoFe2O4 nanorods-PANI of different feed compositions: (a) and (b), rS = 6; (c) and (d), rS = 12; (e) rS = 20; (f), (g) and (h), rS = 16. In image pairs (a) - (b), (c) - d), and (g) - (h) the same region of the sample was imaged with two different detectors: InLens in and QBSD, respectively. The same particle is encircled in each case for better comparison.

$100 \times 75 \mathrm{~mm}(150 \times 150 \mathrm{DPI})$ 


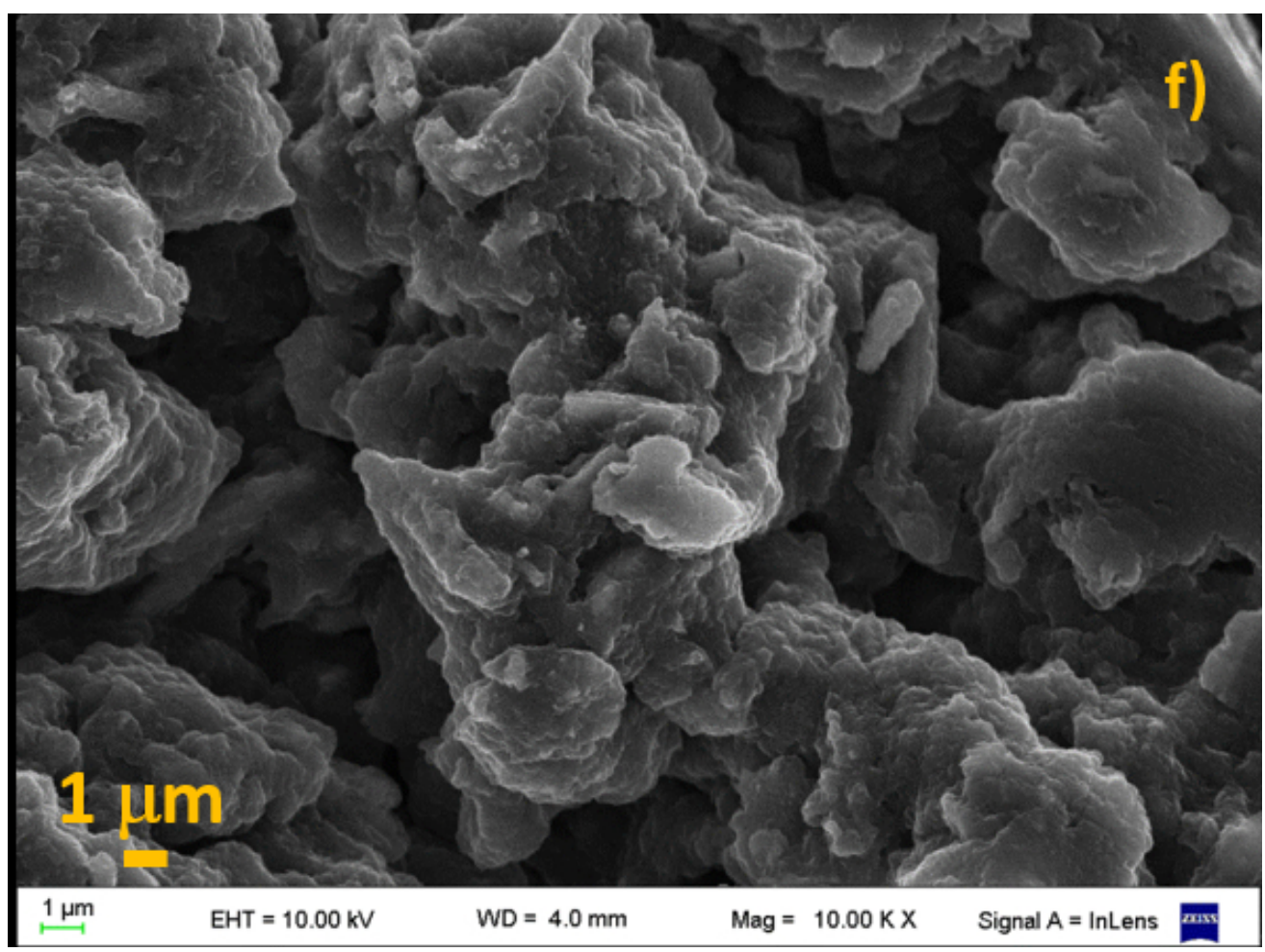

FIG. 8. Typical SEM images of CoFe2O4 nanorods-PANI of different feed compositions: (a) and (b), rS = 6; (c) and (d), rS = 12; (e) rS = 20; (f), (g) and (h), rS = 16. In image pairs (a) - (b), (c) - d), and (g) - (h) the same region of the sample was imaged with two different detectors: InLens in and QBSD, respectively. The same particle is encircled in each case for better comparison.

$$
100 \times 75 \mathrm{~mm}(150 \times 150 \mathrm{DPI})
$$




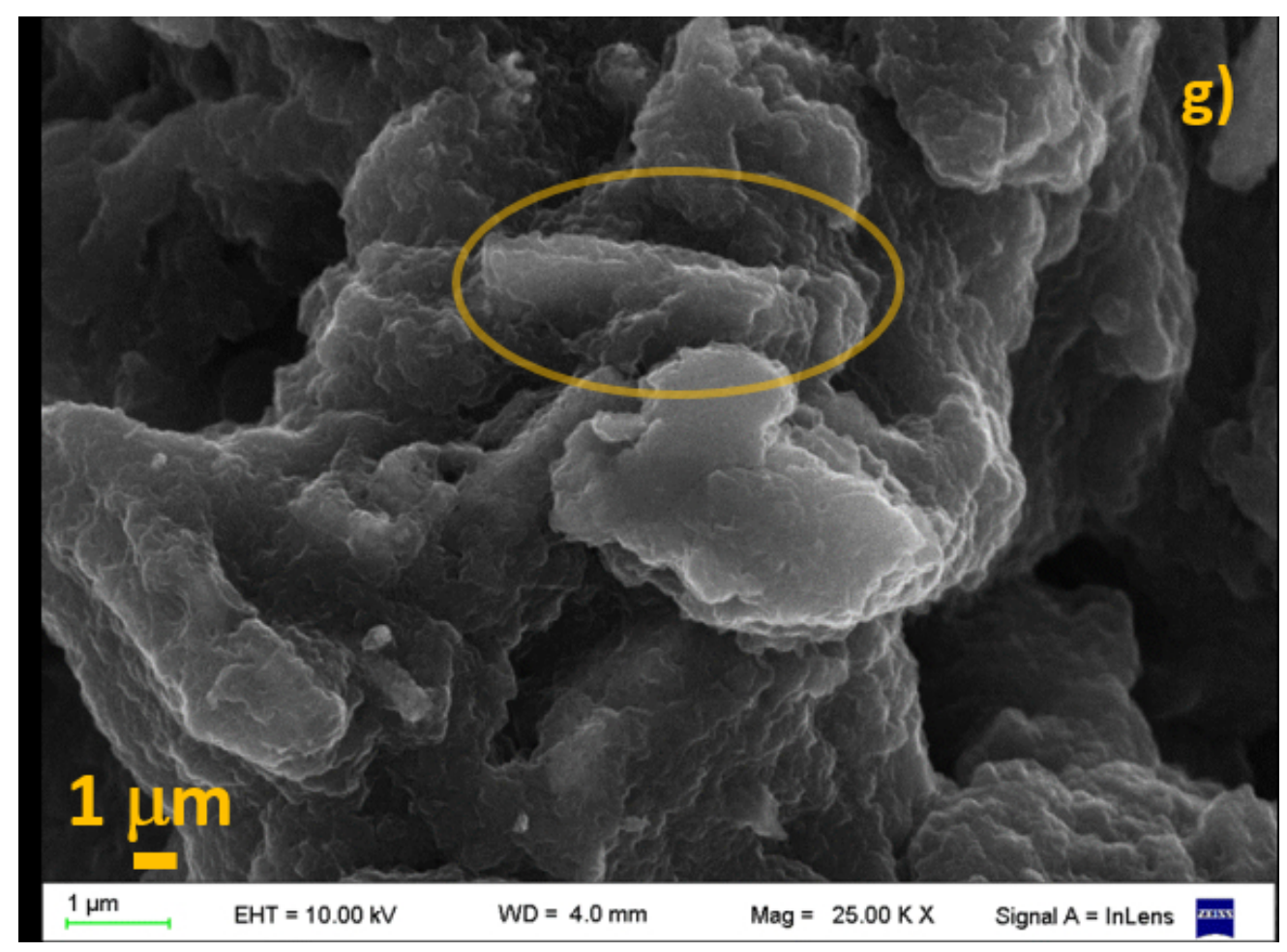

FIG. 8. Typical SEM images of CoFe2O4 nanorods-PANI of different feed compositions: (a) and (b), rS = 6; (c) and (d), rS = 12; (e) rS = 20; (f), (g) and (h), rS = 16. In image pairs (a) - (b), (c) - d), and (g) - (h) the same region of the sample was imaged with two different detectors: InLens in and QBSD, respectively. The same particle is encircled in each case for better comparison.

$$
101 \times 75 \mathrm{~mm}(150 \times 150 \mathrm{DPI})
$$




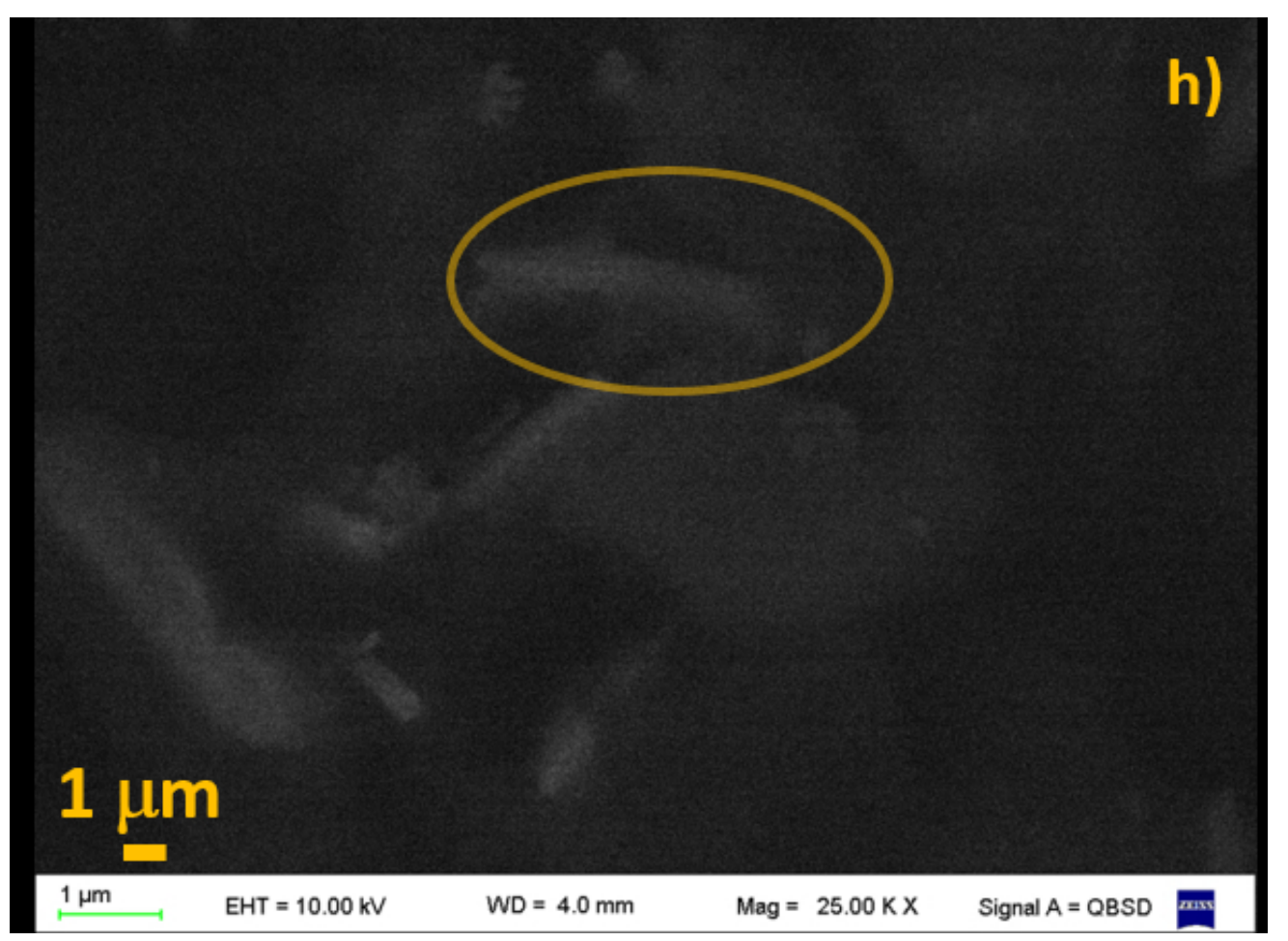

FIG. 8. Typical SEM images of CoFe2O4 nanorods-PANI of different feed compositions: (a) and (b), rS = 6; (c) and (d), rS = 12; (e) rS = 20; (f), (g) and (h), rS = 16. In image pairs (a) - (b), (c) - d), and (g) - (h) the same region of the sample was imaged with two different detectors: InLens in and QBSD, respectively. The same particle is encircled in each case for better comparison.

$$
102 \times 75 \mathrm{~mm}(150 \times 150 \mathrm{DPI})
$$




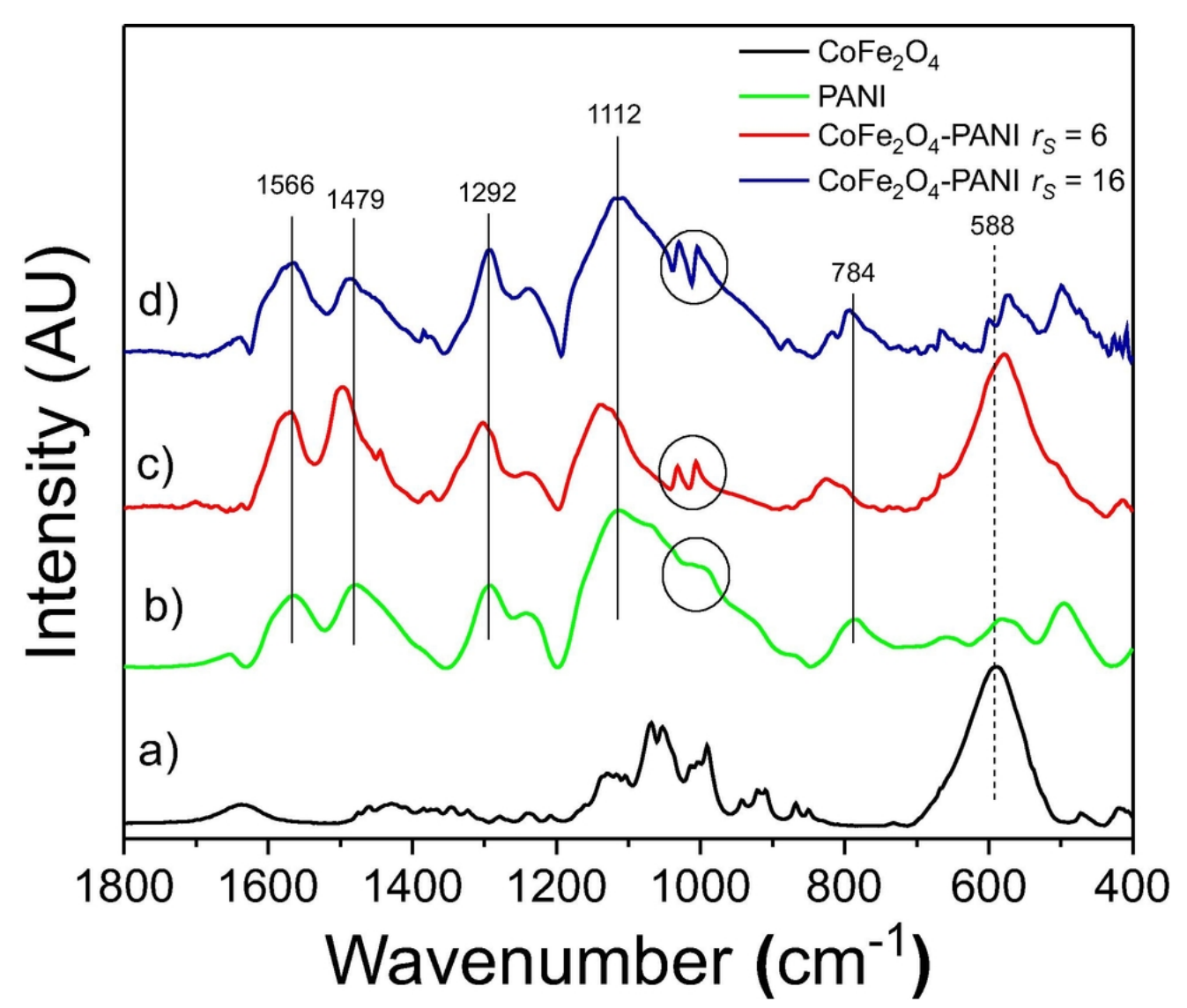

FIG. 9. IR spectra for the $1800-400 \mathrm{~cm}-1$ region of samples of: a) CoFe2O4 3.0\% PVA, b) pure PANI, and CoFe2O4-PANI composites c) $r S=6$ and d) $r S=16$.

$82 \times 70 \mathrm{~mm}(300 \times 300$ DPI $)$ 


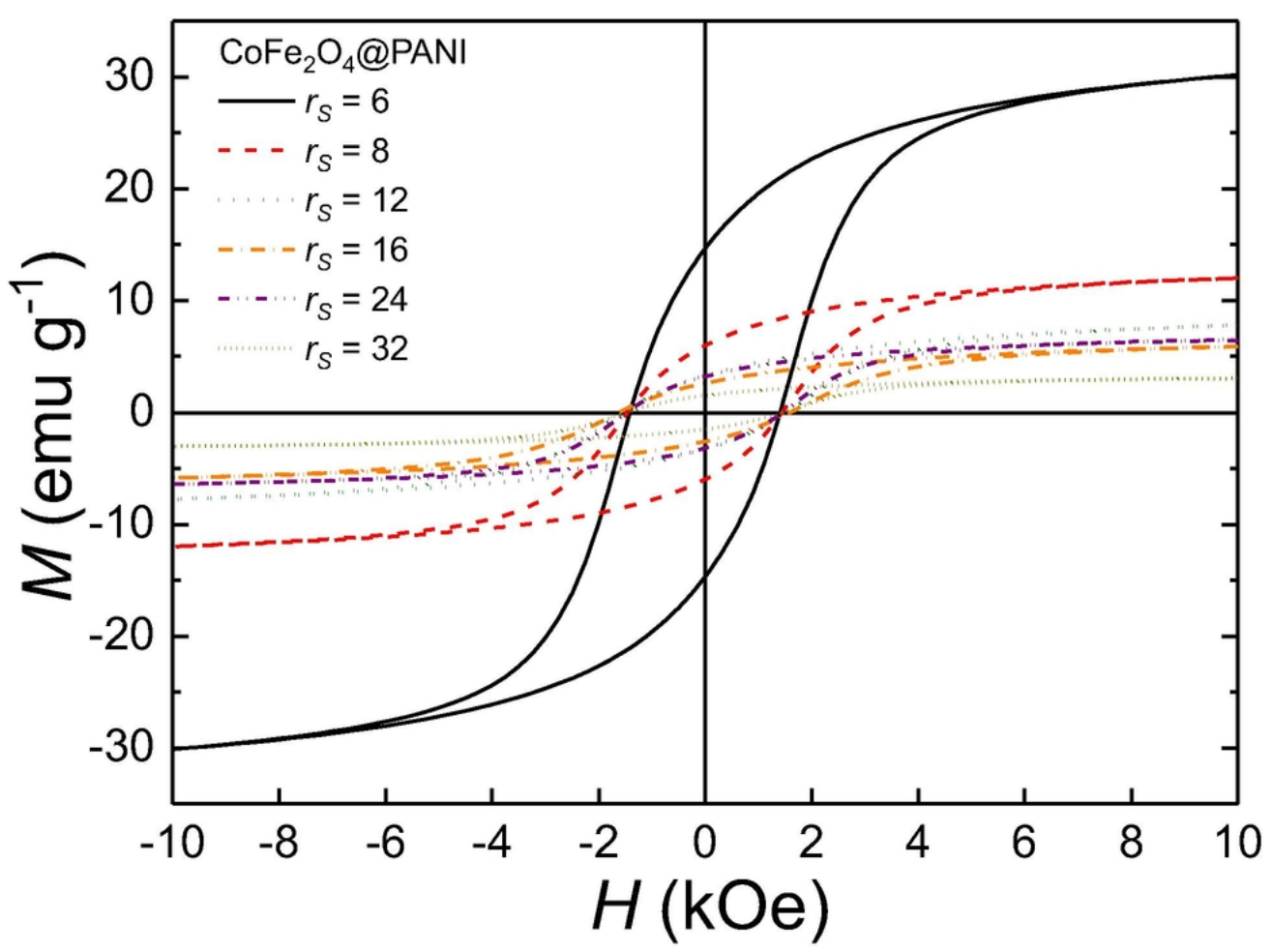

FIG. 10. Magnetization curves for CoFe2O4-PANI composites for different rS values: (a) relative to the total mass of the composite (b) relative to the mass of cobalt ferrite within the composite sample.

$82 \times 63 \mathrm{~mm}(300 \times 300 \mathrm{DPI})$ 


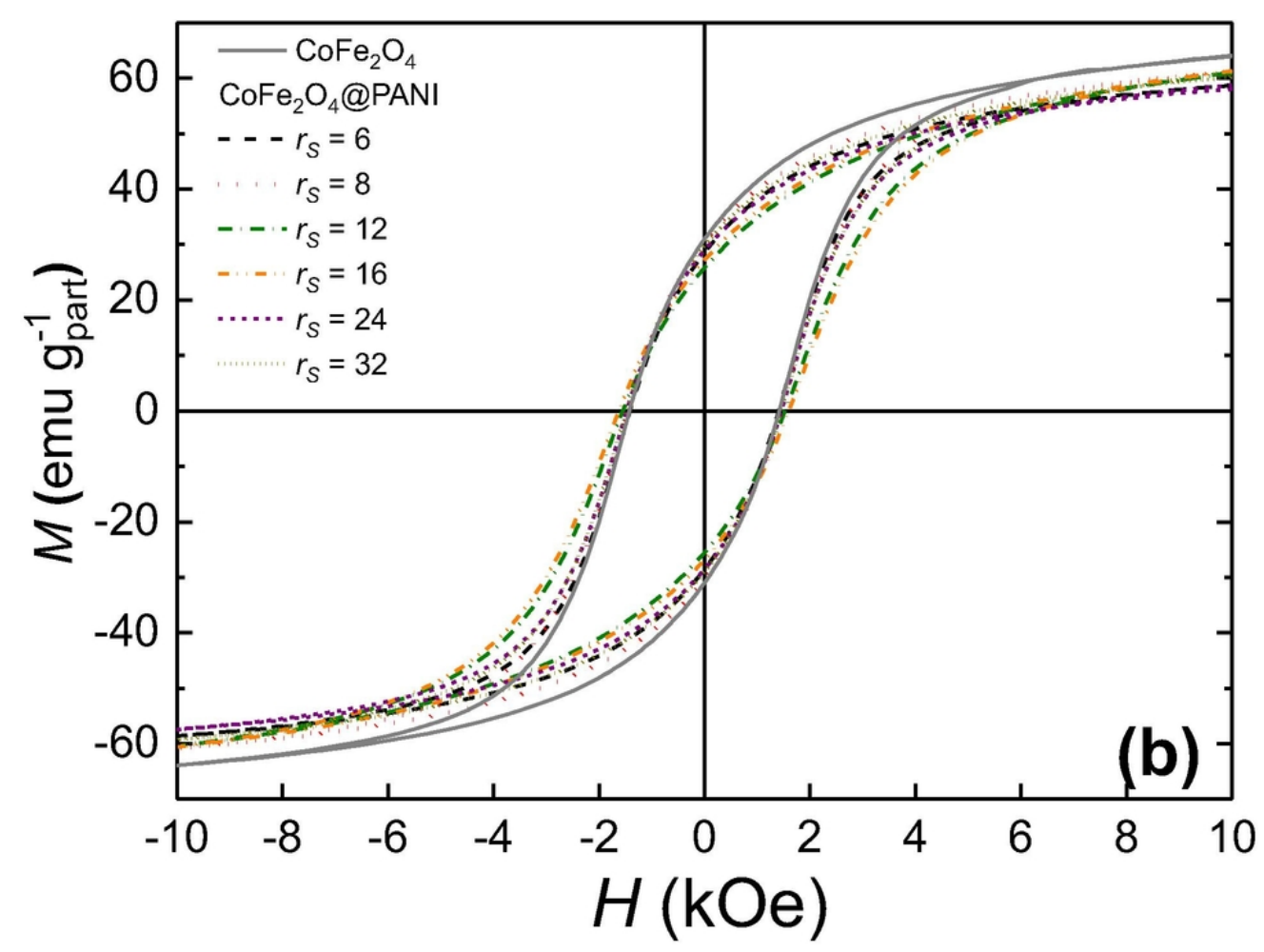

FIG. 10. Magnetization curves for CoFe2O4-PANI composites for different rS values: (a) relative to the total mass of the composite (b) relative to the mass of cobalt ferrite within the composite sample.

$81 \times 61 \mathrm{~mm}(300 \times 300 \mathrm{DPI})$ 